\title{
Mehlem, Ulrich; Erdogan, Ezgi
}

\section{Kategoriensystem zur Kodierung der interaktiven Einbettung sprachlicher}

\section{Beiträge von Kindern in schulischen und vorschulischen}

\section{Sprachförderaktivitäten}

Mackowiak, Katja [Hrsg.]; Beckerle, Christine [Hrsg.]; Gentrup, Sarah [Hrsg.]; Titz, Cora [Hrsg.]: Forschungsinstrumente im Kontext institutioneller (schrift-)sprachlicher Bildung. Bad Heilbrunn : Verlag Julius Klinkhardt 2020, S. 121-140

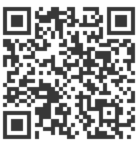

\section{Quellenangabe/ Reference:}

Mehlem, Ulrich; Erdogan, Ezgi: Kategoriensystem zur Kodierung der interaktiven Einbettung sprachlicher Beiträge von Kindern in schulischen und vorschulischen Sprachförderaktivitäten - In: Mackowiak, Katja [Hrsg.]; Beckerle, Christine [Hrsg.]; Gentrup, Sarah [Hrsg.]; Titz, Cora [Hrsg.]:

Forschungsinstrumente im Kontext institutioneller (schrift-)sprachlicher Bildung. Bad Heilbrunn : Verlag Julius Klinkhardt 2020, S. 121-140 - URN: urn:nbn:de:0111-pedocs-201475 - DOI: 10.25656/01:20147

https://nbn-resolving.org/urn:nbn:de:0111-pedocs-201475

https://doi.org/10.25656/01:20147

in Kooperation mit / in cooperation with:

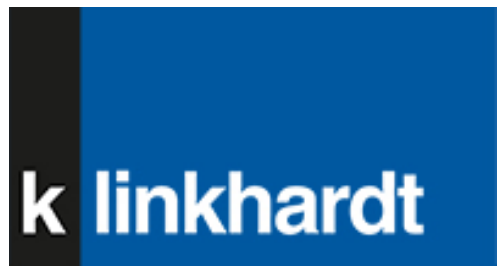

http://www.klinkhardt.de

\section{Nutzungsbedingungen}

Dieses Dokument steht unter folgender Creative Commons-Lizenz: http://creativecommons.org/licenses/by-nc-sa/4.0/deed.de - Sie dürfen das Werk bzw. den Inhalt unter folgenden Bedingungen vervielfältigen, verbreiten und öffentlich zugänglich machen sowie Abwandlungen und Bearbeitungen des Werkes bzw. Inhaltes anfertigen: Sie müssen den Namen des Autors/Rechteinhabers in der von ihm festgelegten Weise nennen. Dieses Werk bzw. der Inhalt darf nicht für kommerzielle Žwecke verwendet werden. Die neu entstandenen Werke bzw. Inhalte dürfen nur unter Verwendung von Lizenzbedingungen weitergegeben werden, die mit denen dieses Lizenzbedingungen weitergegeben werden,

Mit der Verwendung dieses Dokuments erkennen Sie die Nutzungsbedingungen an.

\section{Terms of use}

This document is published under following Creative Commons-License: http://creativecommons.org/licenses/by-nc-sa/4.0/deed.en - You may copy, distribute and transmit, adapt or exhibit the work in the public and alter, transform or change this work as long as you attribute the work in the manner specified by the author or licensor. You are not allowed to make commercial use of the work. If you alter, transform, or change this work in any way, you may distribute the resulting work only under this or a comparable license.

By using this particular document, you accept the above-stated conditions of use.

\section{Kontakt / Contact:}

\section{peDOCs}

DIPF | Leibniz-Institut für Bildungsforschung und Bildungsinformation Informationszentrum (IZ) Bildung

E-Mail: pedocs@dipf.de

Internet: www.pedocs.de

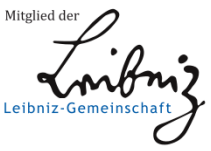




\section{Ulrich Mehlem und Ezgi Erdogan}

\section{Kategoriensystem zur Kodierung der interaktiven Einbettung sprachlicher Beiträge von Kindern in schulischen und vorschulischen Sprachförderaktivitäten}

\section{Info-Kasten zum Instrument}

$\begin{array}{ll}\text { Name } & \begin{array}{l}\text { Kategoriensystem zur Kodierung der Form der interaktiven Einbet- } \\ \text { tung sprachlicher Beiträge von Kindern in Sprachfördersettings }\end{array} \\ \text { Autor*innen } & \begin{array}{l}\text { Ulrich Mehlem und Ezgi Erdogan } \\ \text { Zestimmung der Anzahl spontaner, selbstinitiierter Beiträge von aus- } \\ \text { gewählten Kindern in Sprachfördersettings, woraus sich Rückschlüsse } \\ \text { auf die Offenheit oder Lenkung der Interaktionssituation ziehen lassen }\end{array} \\ \text { Einsatzbereich } & \begin{array}{l}\text { Kleingruppensituationen in Kita und Grundschule } \\ \text { Erhebung }\end{array} \\ \text { Auswertung } & \begin{array}{l}\text { Quantitatives Kategoriensystem } \\ \text { Quanien mit Transkription }\end{array}\end{array}$

\section{Theoretischer Hintergrund zum Instrument}

Das Instrument erfasst Gesprächsbeiträge einzelner Kinder in KleingruppenInteraktionen und systematisiert diese nach der Form ihrer Einbettung in den Interaktionsverlauf. Im Fokus steht die Unterscheidung zwischen Beiträgen, die vom Kind selbst initiiert werden, und solchen, die an Impulse der Fachkraft oder anderer Kinder anschließen. Einer solchen kindorientierten Analyseperspektive liegt die These zugrunde, dass ein höherer Anteil an vom Kind initiierten Beiträgen auch mit einer stärkeren und sprachlich komplexeren Interaktionsbeteiligung der Kinder einhergeht. Ennemoser, Kuhl und Pepouna (2013) weisen eine besondere Wirksamkeit des dialogischen Bilderbuchlesens auf die expressive Sprachkompetenz der geförderten Kinder nach. Sie nennen auf Fachkraftseite unter Verstärkung/Motivation auch die Orientierung an Interessen und Erfahrungen des Kindes als sprachförderliches Interaktionsmerkmal, was entsprechende Äußerungen von Kindern einschließt. Isler, Künzli und Wiesner (2014) arbeiten in ihren Mikroanalysen von bilderbuchbezogenen Interaktionen die Selbstinitiierung, also das Ergreifen sprachlicher Initiative von Kindern, als besondere Lerngelegenheit 
heraus. Auch in den Analysen zu dialogisch-entwickelnden Denkprozessen (sustained shaired thinking) spielen Kind-initiierte Beiträge eine große Rolle (König, 2009; Melhuish, 2013; Siraj-Blatchford, 2009). Das Auftauchen eines Problems, das Kinder zum Nachdenken anregt, kann häufig nicht von der Fachkraft vorher geplant werden, sondern ergibt sich in einem ,fruchtbaren Moment' (Meyer-Drawe, 1984) des Spiels oder der Erkundung, an dem Kinder einen maßgeblichen Anteil haben. Dieses gilt auch für Erzählungen, die nicht nur ,auf Anforderung (Rehbein, 2007), sondern spontan und angeregt durch die Spiel- oder Gesprächssituation entstehen, wenn der hierfür nötige Freiraum gegeben ist. In ihrer Analyse zielkindbezogener sprachlicher Interaktionen von Fachkräften unterscheiden Kannengieser und Tovote (2015) leider nicht zwischen den Gesprächsanteilen von Kindern und Fachkräften, können aber dennoch eine unterschiedliche Sprachförderqualität bestimmter sprachlicher Handlungen aufgrund ihres Kommunikationszwecks ausmachen; hierbei stellt die Multidirektionalität, d.h. das Zusammentreffen unterschiedlicher Perspektiven von Interaktionsteilnehmer*innen, ein entscheidendes Kriterium dar. Schließlich konnten Egert, Quehenberger, Dederer und Wirts (2018) zeigen, dass es sich auf die sprachförderliche Qualität einer Interaktionssituation auswirkt, ob die Aktivität auf die Initiative eines Kindes zurückging. In allen diesen Arbeiten spielt kindliche Initiative eine wichtige Rolle, ohne dass bisher versucht wurde, die Frage zu klären, um welche soziale und sprachliche Aktivität es sich hierbei handelt und in welchem Zusammenhang diese zu Praktiken der Sprachförderung steht.

Die ethnomethodologische Konversationsanalyse (Garfinkel, 1967; Goodwin, 1981; Schegloff, 1996) stellt unseres Erachtens einen geeigneten theoretischen Rahmen für die Klärung dieser Frage dar. Hier kann an eine lange Tradition der Anwendung solcher Verfahren in pädagogischen Kontexten, vor allem auf Unterricht (Becker-Mrotzek \& Vogt, 2009; Lüders, 2003) und auf Gespräche in Familien (Heller, 2012; Morek, 2012) zurückgegriffen werden. Darüber hinaus stützen sich die folgenden Analysen auf linguistische Arbeiten zur gesprochenen Sprache (Chafe, 1994; Maas, 2010; Quasthoff, 2012; Rehbein, 2007). Eine soziale Situation wird danach gemeinsam durch alle Interaktionsteilnehmer*innen hervorgebracht. Indem ihre individuellen Äußerungen als Turns (Gesprächsbeiträge) ständig aufeinander Bezug nehmen, werden Themen konstituiert und sukzessive bearbeitet. Jeder Turn schließt an Vorgängeräußerungen an und löst Reaktionen bzw. Folgeäußerungen aus. Entscheidendes Kriterium für einen Turn ist dabei der Sprecher*innenwechsel. Ein Turn selbst kann aus unterschiedlich vielen kleineren Einheiten (sogenannten ,Turn Constructional Units' TCU, Schegloff, 1996) bestehen, die durch prosodische, informationsstrukturelle und grammatische Kriterien bestimmt werden.

Mit solchen Turns vollziehen die Sprecher*innen interaktive Züge, denen häufig vom/von der Interaktionspartner*in gesetzte Zugzwänge vorausgehen, die einen 
solchen Zug in besonderer Weise erwartbar machen (Heller, 2012; Morek, 2012). Dieses können direkte Fragen oder Aufforderungen z.B. an ein Kind sein, sie können sich aber auch aus einem Ritual oder einem routinierten Handlungsablauf ergeben. Interaktive Züge können aber auch einen hohen Grad an Unabhängigkeit vom interaktiven Kontext aufweisen, wenn es dem/der Sprecher*in gelingt, ein neues Thema mit der bisherigen Interaktion zu verknüpfen.

Die Konversationsanalyse nimmt eine transindividuelle Perspektive auf die soziale Situation ein, bei der es auf die gemeinsame Erledigung interaktiver Aufgaben bzw. Jobs durch die Interaktionsteilnehmer*innen ankommt. Bergmann und Quasthoff (2010) schlagen vor, zusätzlich zu einer solchen Betrachtungsebene eine individuelle Ebene zu untersuchen, in der einzelne Beiträge ihren jeweiligen ,Autor*innen' zugerechnet werden. In dieser Perspektive ist dann auch - neben der grammatischen Struktur - die Form der Einbettung einer Äußerung in ihren Kontext relevant.

Der Grad der individuellen Beteiligung einer Person an einem Gespräch hängt in hohem Maße davon ab, wie ihre Gesprächsbeiträge in den interaktiven Kontext eingebettet sind: Reagiert er/sie nur auf Zugzwänge der Gesprächspartner*innen oder nutzt er/sie Gelegenheiten, seine/ihre Themen zu etablieren und öfter das Rederecht für sich zu reklamieren? Eine längere Forschungstradition konnte am Beispiel von Erzählungen zeigen, welche entscheidende Rolle deren Einbettung in ein Gespräch für ihr Zustandekommen spielt. Fünf unterschiedliche Jobs werden hierbei unterschieden: Durch die Ankündigung einer besonderen Inhalts- oder Formrelevanz (1) werden die Gesprächspartner*innen zunächst auf einen Kontext eingestimmt, in dem die eigentliche Themensetzung (2) nicht mehr willkürlich erscheint. Beide Schritte bedürfen aber der Ratifizierung durch die potenziellen Zuhörer*innen, bevor die Elaboration oder Dramatisierung der Erzählung (3) folgen kÖnnen. Am Ende einer so gerahmten Erzählung stehen dann die Jobs des Abschließens (4) und Überleitens (5) (Hausendorf \& Quasthoff, 2005).

Vor allem in einer spracherwerbstheoretischen Perspektive ist die Kombination der transindividuellen mit der individuellen Perspektive notwendig. Das Übergabeprinzip (Bruner, 1987), wonach erwachsene oder kompetentere Sprecher*innen sukzessive immer mehr interaktive Jobs an die Kinder oder weniger kompetenten Sprecher*innen übertragen, bedeutet für deren individuelle Beiträge, dass diese mit einer immer größeren Sprachkompetenz verbunden sind. Diese kann daher zu einem gewissen Grad aus der zunehmend komplexeren Bearbeitung der interaktiven Jobs extrapoliert werden. So ist es möglich, Äußerungen, die im Rahmen einer gemeinsam konstituierten sozialen Situation zustande kommen (Aktualgenese), gleichzeitig als Performanz individueller Sprachkompetenz zu einem gegebenen Zeitpunkt im Rahmen der Ontogenese zu analysieren (Bergmann \& Quasthoff, 2010). 
Unterschieden werden dabei unterschiedliche kommunikative Gattungen (Heller, 2012). Neben dem Erzählen spielen insbesondere das Erklären und Argumentieren eine wichtige Rolle für die Herausbildung von Diskurskompetenz, also die Hervorbringung satzübergreifender Diskurseinheiten durch einzelne Sprecher*innen, in denen sich eine höhere Sprachkompetenz als in Einzeläußerungen zeigt. Allerdings zeigen Erwerbsstudien, dass jüngere Kinder solche Diskurseinheiten noch nicht von alleine zustande bringen, sondern auf Unterstützung durch kompetente Gesprächspartner*innen angewiesen sind, die einen Teil der interaktiven Jobs für sie übernehmen und ihnen durch lokale Zugzwänge ein schrittweises Bearbeiten der Erzähljobs ermöglichen (Quasthoff, 2012).

Unterrichtsgespräche sind durch eine starke Wissens-Asymmetrie der jeweiligen Gesprächsrollen zulasten der Kinder gekennzeichnet. Die prototypische Abfolge ist lehrergelenkte Initiierung, schülerseitige Antwort und lehrerseitige Bewertung (IRE-Schema: Mehan, 1979; gelenkte Gesprächssituation: vgl. auch Lüders, 2003). Dialoge in der Familie weisen dagegen trotz des Generationenunterschieds eine größere Bandbreite an Stilen und Genres auf; Lehr-Lern-Dialoge treten hier viel seltener auf (Heller, 2012). Dies gilt auch für Interaktionen in Kitas bzw. vorschulischen Settings, in denen die Themenentwicklung nicht allein durch den/die Erwachsene*n gesteuert wird, sondern auch Impulse von den kindlichen Interaktionsteilnehmer*innen erhält (offene Gesprächssituation). Die hier dargestellten Pole der stärker gelenkten gegenüber der offeneren Gesprächssituation weisen auf unterschiedliche sprachliche Beteiligungsmöglichkeiten von Kindern hin.

Etwas holzschnittartig ergibt sich hieraus als Hypothese, dass in offen strukturierten vorschulischen Settings mit der Zahl der selbstinitiierten Beiträge von Kindern auch die sprachliche und syntaktische Komplexität ihrer Beiträge insgesamt zunimmt, während Kinder in stark gelenkten Situationen fast ausschließlich auf Zugzwänge der Fach- bzw. Lehrkraft reagieren, was auch die Komplexität ihrer Beiträge beeinträchtigt. Dagegen wird vermutet, dass das Ergreifen von Gelegenheiten für spontane Äußerungen nicht direkt von der Sprachkompetenz abhängig ist.

Durch den Blick auf die Form der Einbettung kindlicher Beiträge in vorschulischen und schulischen Settings wird die verbreitete Analyseperspektive, die nach den sprachförderlichen Strategien der Fach- und Lehrkräfte fragt (Beckerle, 2017; Kammermeyer et al., 2019; Kucharz, 2018), an einer entscheidenden Stelle ergänzt. Sprachförderliches Handeln von Fach- und Lehrkräften zeigt sich nicht nur an der Häufigkeit bestimmter Frage-, Modellierungs- und Feedbacktechniken, sondern auch darin, ob für die Kinder in der Situation Gelegenheitsstrukturen für Beiträge entstehen, in denen sie selbst die Initiative ergreifen können (Isler, Künzli \& Wiesner, 2014; Kucharz, Mehlem, Rezagholinia \& Erdogan, 2018). Diese bieten dann wiederum Gelegenheiten für Fachkräfte, hieran anzuknüpfen, z.B. 
durch Beantwortung der Fragen von Kindern (Kappauf, 2018). Auch die Zahl der Züge beim sustained shared thinking (Hormann \& Koch, 2014) und das Sprechen von Kindern untereinander (unterstützt durch das Redirect der Fachkraft: Löffler \& Vogt, 2015) weisen auf Gelegenheitsstrukturen für spontane, selbstinitiierte Beiträge von Kindern hin.

Bislang existiert jedoch noch kein geeignetes Instrument, um solche Gelegenheitsstrukturen auch quantitativ durch die Zahl der von Kindern initiierten Gesprächsbeiträge zu bestimmen. Deshalb wurde in der im Rahmen der BISSInitiative (Bildung durch Sprache und Schrift) durchgeführten Evaluationsstudie Sprachförderung am Übergang Kindergarten und Grundschule evaluieren (SPRÜNGE) ein solcher Versuch unternommen.

\section{Vorstellung des Kategoriensystems}

\subsection{Vorarbeiten und Festlegung der Kodiereinheiten}

Bei dem vorgestellten Instrument handelt es sich um ein Kategoriensystem, mit dessen Hilfe die Form der interaktiven Einbettung kindlicher Äußerungen in Sprachfördersettings und ihre syntaktische Struktur kodiert werden können. Es liegt als elektronisches Manual vor (vgl. Online-Anhang). Die Anwendung erfolgt mithilfe von händischen Eintragungen. Sie setzt ein detailliertes Transkript einer videografierten Szene mit EXMARALDA (Schmidt, 2016) oder F4 (F4transkript, 2015) voraus, das sich möglichst an den Konventionen von GAT (Gesprächsanalytisches Transkriptionssystem) orientieren sollte (Selting et al., 1998). In einem solchen Transkript können verbale und relevante nonverbale Beiträge einer Gruppeninteraktion aber auch in einer Partiturschreibweise angeordnet werden, die die sprachlichen Überlappungen und parallele Handlungen auch graphisch anzeigt. Die Weiterverarbeitung der Daten kann dann mit MAXQDA (Kuckartz, 2010) erfolgen, möglich ist aber auch die Verwendung von EXCEL. Das Instrument richtet sich an Forscher*innen, die interaktionsanalytisch arbeiten, aber ihre Ergebnisse zusätzlich quantitativ absichern und mit anderen Kinder- oder Fachkraftdaten triangulieren möchten.

Vor Beginn der Einbettungsanalyse wird in Abhängigkeit von der zu untersuchenden Fragestellung entschieden, welche Kinder im jeweiligen Setting genauer untersucht werden sollen, also Fokuskinder darstellen. Dies können sprachlich besonders aktive oder besonders zurückhaltende Kinder sein. Aussagen über die Interaktionsqualität eines Settings sollten immer auf der Analyse mehrerer Kinder aufbauen. Dagegen ist die Analyse der Einbettungsformen aller Beiträge aller Kinder nicht notwendig. Gegebenenfalls können auch Kinder mit besonderem Sprachförderbedarf fokussiert werden. 
Im Folgenden werden die Kategorien des Instruments mithilfe von Daten erläutert, die innerhalb der Evaluationsstudie SPRÜNGE erhoben wurden. Hierbei handelte es sich um ein längsschnittlich und mehrperspektivisch angelegtes Forschungsprojekt, das darauf abzielte, praktizierte Sprachfördermaßnahmen hinsichtlich ihrer Wirksamkeit im letzten Kitajahr bis zum ersten Schuljahr der Grundschule zu erforschen. Dabei wurden vier Dimensionen der Sprachförderung am Übergang Kita-Grundschule näher beleuchtet: 1. die Sprachentwicklung einer Kinderkohorte und 2. die Sprachförderkompetenz der Fach- und Lehrkräfte zu drei Messzeitpunkten (MZP), 3. die Interaktionsstrukturen von sprachförderlichen Aktivitäten und 4. Orientierungen der Fach- und Lehrkräfte beim Sprachförderhandeln und der Kooperation (Erdogan et al., im Druck; Kucharz et al., 2018). In diesem Beitrag geht es um das Verfahren, welches zur Analyse der Interaktionsstrukturen in den Sprachfördersettings angewendet wurde. Hierbei wird auf die Transkriptauszüge des Anhangs verwiesen. Weitere Ankerbeispiele und Erklärungen befinden sich ebenfalls im Anhang (vgl. Online-Anhang).

Die Einbettungsanalyse verlangt zunächst die Bestimmung der relevanten Kodiereinheit; dies ist jeweils ein Turn eines*r Sprechers*in im Sinne der oben gegebenen Definition. Eine Äußerung wird auch dann als einziger Turn kodiert, wenn sie durch Interjektionen unterbrochen wird oder mit anderen Äußerungen überlappt, solange kein definitiver Sprecher*innenwechsel eintritt. Bei Turns, die aus mehreren Konstituenten bestehen, werden diese für die Einbettungsanalyse nicht getrennt berücksichtigt, da sie jeweils als Ganzes den Gesprächsbeitrag ausmachen (Schegloff, 1996). Konstituenten eines Turns (einen Turn konstituierende Einheiten, Turn constituting units = TCU) sind sprachliche Einheiten, die aufgrund von prosodischen und/oder grammatischen Merkmalen deutlich voneinander abgegrenzt werden können. Im Transkriptauszug Lernwerkstatt (VI) tauschen sich mehrere Kinder und die Fachkraft über ,Haie in der Nordsee aus. Die Äußerung von K2 „[es gibt auch] BAbyhaie. es [gibt] glaub ich (.) SÜSShaie“ (VI, 554-557) ${ }^{1 \text { “ }}$ besteht aus zwei Sätzen, die aber nur einen Turn darstellen, da es dazwischen, außer dem Zustimmungssignal der Fachkraft, keinen Sprecher*innenwechsel gibt. Bei längeren, satzübergreifenden Diskurseinheiten eines einzelnen Kindes wird jede weitere satzförmige kindliche Äußerung genauso wie die Äußerung kategorisiert, mit der der kindliche Redebeitrag eröffnet wurde. Dies gilt z.B. bei Erzählungen wie im Transkriptauszug Morgenkreis (IV, 17f.) Für die syntaktische Analyse müssen dagegen alle einen Turn konstituierenden Einheiten jeweils für sich betrachtet werden.

1 In den Verweisen bezieht sich die römische Ziffer jeweils auf die Nummer des Transkriptauszugs, die arabische auf den Turn. 


\subsection{Die Einbettungsanalyse}

In einem zweiten Schritt werden dann die unmittelbaren Kontexte des Turns bestimmt. Im Sinne der ,Next Turn Proof Procedure‘ (Sacks, Schegloff \& Jefferson, 1974) schließt jeder Turn an eine Vorgängeräußerung an, deren interaktive Qualität erst genau durch ihn bestätigt wird, wie umgekehrt erst durch die kommunikativen Anschlüsse an einen Turn dessen Verständnis durch die Teilnehmer*innen selbst gesichert ist.

Bei jedem Turn eines Kindes wird zunächst danach unterschieden, ob er dem Zugzwang eines anderen Interaktionsteilnehmers*in folgt (Fälle 1-4) oder ob er nur lose mit dem vorherigen Interaktionsgeschehen verbunden ist und damit als kindliche Initiative bzw. Selbstinitiierung bestimmt werden kann (Fall 5).

Bei den unterschiedlichen Formen von Zugzwängen geht es um die Folgenden:

1. Ein Zugzwang, der durch gemeinsames sprachliches Handeln der gesamten Gruppe entsteht und vom Kind gemeinsam mit anderen Kindern bedient wird, wie z.B. das Mitsingen eines Liedes oder der sprachliche Vollzug eines gemeinsamen Rituals. So spricht im Transkript V Kind 1 (K1) parallel zur Fachkraft und den anderen Kindern einen Begrüßungsvers mit: „, [alle kinder sind jetz fit bist du's AUCH? dann Mach gleich MIT]“ (V, 6-7)

2. Zugzwang mit direkter Rederechtserteilung: Fragen bzw. Aufforderungen, welche die Fachkraft direkt an ein bestimmtes Kind richtet oder die in routinierten Handlungsstrukturen entstehen (z.B. An-die-Reihe-Kommen in einer Spielaktivität). Die direkte Ansprache eines Kindes erfolgt etwa durch Namensnennung seitens der Fachkraft (vgl. V/44) oder durch eine entsprechende Geste (Kopfbewegung, Zuwendung oder Blickrichtung). In stärker formalisierten Settings, in denen die Rederechtserteilung schon durch Melden organisiert wird, ist mit der direkt an ein Kind gerichteten Frage der Fachkraft bereits das Rederecht erteilt, sodass das Kind direkt antworten und damit den Zugzwang vollziehen kann.

3. Indirekter Zugzwang ohne direkte Rederechtserteilung: Die Frage bzw. Aufforderung der Fachkraft richtet sich an die gesamte Kindergruppe. Jedes Kind hat die Möglichkeit kommunikativ anzuschließen. In formelleren Settings wird erwartet, dass die Kinder sich melden. Diese nonverbale Aktivität stellt bereits den ersten Zug in der Bedienung des Zugzwangs dar, der aber erst dann berücksichtigt wird, wenn die Fachkraft danach tatsächlich das Rederecht erteilt. Im Transkriptauszug ,Zuckerwürfel' stellt die offene Frage der Fachkraft „was passiert wenn ich den tropfen hier ranmache?" (VII, 328) einen indirekten Zugzwang für alle Kinder am Tisch dar, da niemand speziell angesprochen wird. K2 vollzieht diesen Zugzwang mit der Antwort „das färbt sich“ (VII, 329), ohne dass hierfür eine individuelle Rederechtserteilung erfolgt. Die unmittelbar folgende Nachfrage der Fachkraft (VII, 330) setzt wieder einen indirekten Zugzwang, der diesmal von K1 (VII, 331) 
bearbeitet wird. Bei K4 (VII, 337) ist zu beachten, dass der Zugzwang der Fachkraft, auf den das Kind reagiert, bereits zwei Äußerungen früher (VII, 334) gesetzt wurde.

4. Zugzwänge, die durch ein anderes Kind etabliert werden, treten in formellen Sprachfördersettings wesentlich seltener auf als in informellen. Beim gemeinsamen Spielen kommt es aber häufig zu solchen Interaktionen. In diesem Fall wird nicht zwischen direkten oder indirekten Zugzwängen unterschieden. In der Lernwerkstatt stellt die Frage von K3 „Darf ich mal SEhen, den MEEresschmetterling (VI, 545)?" einen Zugzwang für K2 dar; dieser wird allerdings nonverbal (durch Zeigen) bedient.

5. Den vier durch vorausgehende Zugzwänge charakterisierten Einbettungsformen stehen die selbstinitiierten Beiträge eines Kindes gegenüber. Als solche werden Äußerungen kodiert, wenn sie keinen indirekten bzw. direkten Zugzwang einer Fachkraft oder eines anderen Kindes bedienen. Ihre Einbettung in die Interaktion ist durch höhere Freiheitsgrade gekennzeichnet. Dieses gilt sowohl für die Inanspruchnahme des Rederechts wie für den thematischen Zusammenhang mit dem vorherigen Interaktionsgeschehen. Als Beispiele für selbstinitiierte Äußerungen können die Turns von K3 (VII, 339 „Spongebob Schwammkopf") und der aus zwei Einheiten bestehende Turn von K2 (VII, 340 „aber man kann/aber MANCHmal wenn man zu viel von die FARbe nimmt dann taucht das nach UNten") dienen. Bei K3 lässt sich als Auslöser das Wort ,Schwamm' in der Äußerung der Fachkraft ausmachen. Diesen assoziiert der Junge mit dem Protagonisten der Zeichentrickserie, womit er einen Bezug des Experiments zu seiner lebensweltlichen Erfahrung herstellt. K3 nimmt damit eine thematische Verschiebung vor, die von der Erklärsituation im engeren Sinne wegführt. Dagegen formuliert K2 einen durch ,aber' eingeleiteten Einwand gegenüber der Äußerung der Fachkraft, übernimmt also selbst den Job einer Elaboration im Rahmen einer Erklärung. Er führt seine erste Turnkonstruktionseinheit (TCU) „aber man kann..." nicht zu Ende, sondern repariert sie mit einem sofort nachgeschobenen zweiten TCU, der in Form eines komplexen Satzes einen anderen Zusammenhang zwischen der Farbe und dem Aufsaugen des Zuckerwürfels formuliert. K2 schließt nicht an die Äußerung von K3 an, die damit für das weitere interaktionale Geschehen folgenlos bleibt. Beide Äußerungen lassen sich als Kommentare zu einem Satz der Fachkraft lesen, der selbst - im Sinne der Sprachförderstrategien - eine semantische Expansion darstellt und damit indirekt einen Anlass für weitere Äußerungen liefert.

\subsection{Thematische und syntaktische Analysen}

Neben der interaktionalen Dynamik ist für die Unterscheidung selbstinitiierter Äußerungen von solchen, die Zugzwänge anderer bedienen, auch die thematische 
Organisation des Gesprächs relevant. Selbstinitiierte Äußerungen sind thematisch häufig weniger eng mit dem Vorhergehenden verknüpft (Schegloff, 1996). Entweder stellen sie tatsächlich neue Themensetzungen durch das Fokuskind dar, oder sie kommentieren einen anderen Beitrag, indem Bezüge zu eigenen Erlebnissen des Kindes oder eigene Beobachtungen oder Gedanken mitgeteilt werden. Diese können auch durch weiter zurückliegende Aktivitäten - auch der Fachkraft - ausgelöst worden sein, ohne dass sie tatsächlich interaktive Zugzwänge darstellen. Charakteristisch für selbstinitiierte Äußerungen sind auch Formulierungen von Wünschen, von Widerspruch oder Protest z.B. gegen eine Entscheidung der Fachkraft oder das Verhalten eines anderen Kindes.

Bei den im Transkript VII ,Zuckerwürfel' (Online-Anhang) wiedergegebenen Kinderäußerungen sind zwei von neun selbstinitiiert: bei K2 eine selbstinitiierte Äußerung gegenüber vier Reaktionen auf Zugzwänge, bei K3 je eine selbstinitiierte und eine Reaktion auf einen Zugzwang.

Wie bereits im Theorieteil ausgeführt, wird in der Übernahme zusätzlicher interaktiver Jobs eine treibende Kraft der Sprachentwicklung vermutet (Quasthoff, 2012). Es wird angenommen, dass hiermit auch eine Zunahme der Fähigkeit, sich situationsangemessen, differenziert und syntaktisch vollständig zu äußern, verbunden ist (diskursive Kompetenz im Sinne von Ehlich, Bredel \& Reich, 2008). Dies bedeutet allerdings nicht, dass sich die Satzstruktur selbstinitiierter Äußerungen grundsätzlich von Äußerungen unterscheidet, die auf Zugzwänge antworten. Einwortäußerungen gehen zwar häufig auf Zugzwänge zurück (,nein' bei einer Entscheidungsfrage, Nennung eines Nomens bei einer Ergänzungsfrage), können aber auch global und damit sehr umfassend bedient werden. Umgekehrt sind bei selbstinitiierten Äußerungen neben „ich kann auch die OHREN machen“ (Kucharz et al. 2018, S. 191) auch sehr knappe Turns („das will ich“ bzw. „gib mal“ als Ausdruck eines Wunsches) in unseren Daten belegt.

Ergänzend zur Einbettung wird daher in unserem Kategoriensystem eine Analyse der syntaktischen Komplexität durchgeführt, wobei das entscheidende Kriterium die Satzförmigkeit der Äußerung darstellt. Auf dieser Grundlage kann dann untersucht werden, in welchem Grad Selbstinitiierung und Satzförmigkeit korrelieren. Von den Kinderäußerungen im Transkript VII ,Zuckerwürfel‘ sind zwei Äußerungen satzförmig, eine bedient einen Zugzwang, die andere ist selbstinitiiert; diese enthält auch zwei Teilsätze, die doppelt gezählt werden.

\subsection{Quantifizierung und Vergleichbarkeit}

Die Bestimmung der Anzahl selbstinitiierter Äußerungen bezieht sich zunächst auf einzelne Kinder, die zu unterschiedlichen Zeitpunkten ihrer Förderung oder in Abhängigkeit vom Sprachstand in den Blick genommen werden können. Sie kann aber auch für ein ganzes Sprachfördersetting angegeben werden. Dann soll- 
ten aber Daten von mehr als einem Kind (nicht zwangsläufig von allen) berücksichtigt werden.

Um Vergleichbarkeit zu ermöglichen, wird die Anzahl der selbstinitiierten Äußerungen pro Minute angegeben. Da in den Settings selten die gleiche Anzahl von Kindern anzutreffen ist, musste außerdem ein Gewichtungsfaktor für die Anzahl an Äußerungen im Verhältnis zur Gruppengröße des Settings (Erdogan et al., im Druck) festgelegt werden. Wenn unterstellt werden kann, dass sich der Redeanteil der Fachkraft durch die Kinderzahl nur unwesentlich ändert (Knapp, Ricart Brede, Gasteiger-Klicpera \& Kucharz, 2009), teilen sich die Kinder den verbliebenen Redeanteil im Durchschnitt gleich. In den bei SPRÜNGE untersuchten Settings kamen am häufigsten fünf Kinder als Gruppengröße vor. Diese Zahl wurde daher als Default-Kinderzahl festgelegt. Beim Gewichtungsfaktor wird die tatsächliche Kinderzahl des Settings durch diese Default-Kinderzahl dividiert. Bei einem Setting mit zwei Kindern (wie in 2.1, vgl. Tab. 4), beträgt dieser Gewichtungsfaktor daher 2/5 (0.4), bei 13 Kindern (wie in 2.3) dagegen 13/5 (2.6). Zehn selbstinitiierte Äußerungen eines Kindes in Setting 2.1 werden daher nur wie vier Äußerungen gewertet, während die gleiche Zahl Äußerungen in Setting 2.3 wie 26 Äußerungen zählen.

\section{Erprobung des Instruments}

Das Kategoriensystem wurde in der Evaluationsstudie SPRÜNGE entwickelt und eingesetzt. Die Datengrundlage bilden Videoaufzeichnungen, die überwiegend in vorschulischen Sprachfördersettings erhoben wurden. Das Kategoriensystem ist ein Nebenprodukt einer Vielzahl von Interaktionsanalysen, die innerhalb der Forscher*innengruppe diskutiert, überarbeitet und adaptiert wurden. Den Ausgangspunkt bildeten kleinere Szenen von maximal fünf Minuten Dauer, die nach der Lektüre des Transkripts eines vollständigen Settings zur genaueren Analyse ausgewählt wurden. Kriterium für diese Auswahl ist das Auftreten satzübergreifender Diskurseinheiten bei Kindern. Von verschiedenen Mitarbeiter*innen wurden Interaktionsanalysen (Memos) von Ausschnitten solcher Szenen erstellt, die auf der Projektsitzung besprochen wurden. Der gemeinsame Bezug zur Handlungsabfolge im Video diente zur Prüfung der im Memo vorgestellten Interpretation. Die Ergebnisse dieser Diskussionen wurden anschließend in die jeweiligen Memos eingearbeitet. Eine solche Vorgehensweise stellt eine kommunikative Validierung im Sinne eines qualitativen Forschungsparadigmas (Flick, 2019) dar. Das Kategoriensystem wurde entwickelt, um mit einem kleineren und leichter handhabbaren Set von Codes eine größere Datenmenge bearbeiten zu können. Für die Vergabe der Codes der Einbettungsanalyse wurde exemplarisch die Interraterreli- 
abilität berechnet. Das Ergebnis (Cohens Kappa $=0.64, p=0.00$ ) entspricht einer Auswertungsobjektivität im mittleren Bereich.

Insgesamt wurden Daten von 30 Kindern in zehn Settings zu zwei Messzeitpunkten untersucht. In den meisten Settings war auch beim zweiten Messzeitpunkt dieselbe Fachkraft beteiligt; auch der Ablauf war sehr ähnlich. Für fast jedes Kind liegen also Daten zu zwei Messzeitpunkten vor. Unterschieden wurden zunächst additive und alltagsintegrierte Settings. Diese Unterscheidung wurde von den BiSS-Verbünden vorgenommen, deren Arbeit im Rahmen von SPRÜNGE evaluiert wurde. Jeder BiSS-Verbund besteht aus drei bis zehn Kitas und/oder Schulen. Drei Verbünde beschreiben ihre Arbeit als Kombination von additiver und alltagsintegrierter Sprachförderung, wobei in SPRÜNGE nur die additiven Sprachfördersettings untersucht wurden (Kieferle, Reichert-Garschhammer \& Becker-Stoll, 2013, Settings 1-3). Die Zielgruppe sind ausgewählte Kinder im Vorschulalter mit diagnostiziertem Sprachförderbedarf ${ }^{2}$, die in einer Kleingruppe räumlich getrennt gefördert werden. In den anderen BiSS-Verbünden, die im SPRÜNGE-Projekt untersucht wurden, wurden alltagsintegrierte Formen von Sprachförderung durchgeführt, bei denen Kinder mit unterschiedlichem sprachlichen Ausgangsniveau beteiligt waren. In diesen BiSS-Verbünden wurden zur Sprachförderung am Übergang zwischen Kita und Grundschule sogenannte Lernwerkstätten durchgeführt. Es handelte sich um unterschiedliche Formen von Lernwerkstätten (Hagstedt \& Krauth, 2014), einerseits mit Bezügen zum Schriftspracherwerb (Settings 4-7), andererseits zum sachbezogenen Lernen. Hier wurden in der Schule oder einer speziellen Lernwerkstatt durchgeführte Experimente im Anschluss in der Kita nachbereitet (Settings 8-10).

Die folgende Darstellung behandelt zunächst Zusammenhänge von Selbstinitiierung und syntaktischer Komplexität (1), Unterschiede zwischen sprachlich auffälligen und nicht auffälligen Kindern (2) und Unterschiede zwischen additiven und alltagsintegrierten Settings (3).

\subsection{Selbstinitiierung und syntaktische Komplexität}

In einem ersten Schritt werden die Anzahl der für die jeweiligen Kinder ermittelten und nach der Kinderzahl gewichteten selbstinitiierten Äußerungen pro Minute zum ersten und zweiten Messzeitpunkt miteinander und mit der entsprechenden Anzahl der satzförmigen Äußerungen korreliert. 
Tab. 1: Korrelationen selbstinitiierter und satzförmiger Äußerungen

\begin{tabular}{llcccc}
\hline & & SatzMZP1 & iniMZP1 & SatzMZP2 & iniMZP2 \\
\hline \multirow{2}{*}{ SatzMZP1 } & Korr (Pearson) & 1 & $.54^{* *}$ & .34 & -.03 \\
\cline { 2 - 5 } & Sig (2-seitig) & & .00 & .10 & .87 \\
\hline \multirow{2}{*}{ iniMZP1 } & Korr (Pearson) & 1 & $.60^{* *}$ & $.58^{* *}$ \\
\cline { 2 - 5 } & Sig (2-seitig) & & & .00 & .00 \\
\hline \multirow{2}{*}{ SatzMZP2 } & Korr (Pearson) & & 1 & $.61^{* *}$ \\
\cline { 2 - 5 } & Sig (2-seitig) & & & .00 \\
\hline iniMZP2 & Korr (Pearson) & & & & 1 \\
\hline
\end{tabular}

** Die Korrelation ist auf dem Niveau von .01 (2-seitig) signifikant. n MZP1 = 25, MZP2 = 29 Kinder

Zu MZP1 korreliert die Selbstinitiierung signifikant positiv mit der Selbstinitiierung zu MZP2 und mit der Zahl von Sätzen zu beiden MZP. Kinder mit einer stärkeren Tendenz zur Selbstinitiierung im vorletzten Kitajahr behalten diese offenbar auch zu MZP2 bei und produzieren auch später mehr vollständige Sätze. Dagegen korreliert die Zahl der Sätze zu MZP1 nicht signifikant mit MZP2 und sogar negativ mit der Selbstinitiierung zu MZP2. Der Gebrauch einer größeren Zahl von Sätzen zu MZP2 ist also weniger stark vom Satzgebrauch und Selbstinitiierung zu MZP1 abhängig. Ob dies ein Hinweis darauf ist, dass Selbstinitiierung in Verbindung mit vollständigen Sätzen gegen Ende der Kitazeit an Bedeutung verliert, lässt sich aus diesen Daten noch nicht ableiten. Doch sprechen die Befunde dafür, Selbstinitiierung als eigenen Faktor, der sprachlich komplexeren Output begünstigt, weiter zu untersuchen, und zwar im Hinblick darauf, wie sich sprachlich unauffällige von sprachlich auffälligen Kindern unterscheiden.

\subsection{Sprachlich auffällige und unauffällige Kinder im Vergleich}

In einem zweiten Schritt wurden die Daten zu den selbstinitiierten und satzförmigen Kinderbeiträgen mit den Ergebnissen der Sprachstandsfeststellung durch den SET 5-10 (Petermann, 2010) abgeglichen. Bezugsgröße bildete der Gesamtsummenscore über alle Teiltests hinweg, also ein Rohwert (RW). Der SET 5-10 geht (bei fünfährigen Kindern) bei einem Gesamtwert von weniger als 64 Punkten von einem auffälligen Sprachstand aus und leitet daraus einen Förderbedarf ab. Alle Kinder mit einem höheren Rohwert wurden als sprachlich unauffällig eingestuft (Erdogan et al., im Druck). Tabelle 2 zeigt die Mittelwerte der beiden Gruppen für die selbstinitiierten und satzförmigen Äußerungen. 
Tab. 2: Selbstinitiierte und satzförmige Äußerungen bei sprachlich auffälligen und unauffälligen Kindern (SET 5-10)

\begin{tabular}{llll}
\hline & SET 5-10 & $\boldsymbol{n}$ & $\boldsymbol{M}(\boldsymbol{S D})$ \\
\hline \multirow{2}{*}{ satzförmig MZP1 } & auffällig & 8 & $0.47(0.47)$ \\
\cline { 2 - 4 } & unauffällig & 18 & $0.80(0.52)$ \\
\hline \multirow{2}{*}{ satzförmig MZP2 } & auffällig & 11 & $0.56(0.35)$ \\
\cline { 2 - 4 } & unauffällig & 17 & $0.82(0.61)$ \\
\hline \multirow{2}{*}{ selbstinitiiert MZP1 } & auffällig & 8 & $0.74(0.71)$ \\
\cline { 2 - 4 } & unauffällig & 18 & $0.93(0.59)$ \\
\hline \multirow{2}{*}{ selbstinitiiert MZP2 } & auffällig & 11 & $1.01(0.52)$ \\
\cline { 2 - 4 } & unauffällig & 17 & $0.92(0.72)$ \\
\hline
\end{tabular}

Bei den satzförmigen Äußerungen besteht zu MZP1 ein deutlicher Rückstand der auffälligen Kinder ( 0.47 gegenüber 0.80 satzförmigen Äußerungen pro Minute), der zu MZP2 abnimmt. Beim Anteil der selbstinitiierten Äußerungen ist der Abstand schon zu MZP1 deutlich geringer (0.74 gegenüber 0.93) und kehrt sich zu MZP2 sogar zugunsten der sprachlich auffälligen Kinder um (1.01 gegenüber 0.93). Die Mittelwertunterschiede erweisen sich aber beim t-Test als nicht signifikant: dies gilt sowohl für die Satzförmigkeit zu MZP1 $(t(24=-1.27, p=.22)$ und MZP2 $(t(25,16)=-1.38, p=.18)$ als auch bei der Selbstinitiierung (MZP1: $t$ $(24)=-0.99, p=.33$; MZP2: $t(26)=-0.37, p=.72)$. Hierbei ist allerdings zu berücksichtigen, dass die Untersuchungsgruppe relativ klein ist. Die stärkere Zunahme der selbstinitiierten Äußerungen bei den zu MZP1 schwächeren Kindern (im Vergleich mit der Zunahme der Zahl der Sätze) könnte bedeuten, dass dieser Faktor für die Sprachentwicklung an Bedeutung verliert. Es ist aber auch denkbar, dass sich hier Kinder aufgrund ihres Sprachstands zum MZP2 unterschiedlich verhalten.

Kucharz et al. (2018) konnten bei vier Kindern keinen Zusammenhang zwischen dem Sprachverhalten in der Fördersituation und dem gemessenen Sprachförderbedarf finden. Bei dem hier untersuchten Sample zeichnet sich eine Tendenz zu einer größeren Zahl vollständiger Sätze bei den älteren und sprachlich kompetenteren Kindern ab. Der besonders hohe Anteil der selbstinitiierten Äußerungen der zu Beginn sprachauffälligen Kinder zu MZP2 ist damit aber noch nicht erklärt. Es stellt sich damit die Frage nach dem Einfluss des Settings.

\subsection{Additive und alltagsintegrierte Settings}

In einem ersten Schritt werden die Kinderdaten für unterschiedliche Gruppen von Settings differenziert. Dabei werden - entsprechend den in den Verbünden 
praktizierten Konzepten - additive und alltagsintegrierte Sprachfördersituationen unterschieden.

Tab. 3: Sprachlich auffällige und auffällige Kinder, selbstinitiierte und satzförmige Äußerungen nach Settings

\begin{tabular}{llccccc}
\hline & Setting & $\boldsymbol{n}$ Kinder & & $\boldsymbol{n}$ Settings & $\boldsymbol{M}(\boldsymbol{S D})$ \\
\hline & \multicolumn{1}{c}{ auffällig } & unauffällig & & \\
\hline $\begin{array}{l}\text { Satzförmige Äuße- } \\
\text { rungen/pro Kind/pro } \\
\text { Minute }\end{array}$ & additiv & 7 & 1 & 6 & $0.49(0.24)$ \\
\cline { 2 - 7 } & $\begin{array}{l}\text { alltagsin- } \\
\text { tegriert }\end{array}$ & 4 & 18 & 14 & $0.79(0.35)$ \\
\hline $\begin{array}{l}\text { Selbstinitiierte Äuße- } \\
\text { rungen pro Kind/pro } \\
\text { Minute }\end{array}$ & \begin{tabular}{l} 
additiv \\
\cline { 2 - 7 }
\end{tabular} & 7 & 1 & 6 & $0.72(0.31)$ \\
\hline
\end{tabular}

In dieser Tabelle werden zunächst die MZP nicht unterschieden. Die Tabelle zeigt niedrigere Werte der additiven Settings sowohl im Bereich der syntaktische Komplexität als auch der Selbstinitiierung. Bedauerlicherweise sind aber die sprachlich auffälligen und unauffälligen Kinder in den Settings so ungleich verteilt, dass hierbei auch ein Einfluss des Sprachstands wirksam sein könnte. Eine Signifikanzprüfung mithilfe des t-Tests erweist sich auch für diese Gruppenbildung nach Settings als nicht signifikant. Für den jeweiligen Anteil der selbstinitiierten Äußerungen ergibt sich: $t(18)=-1.63, p=.12$, für die Anzahl satzförmiger Äußerungen: $t(18)=-1.87, p=0.08$.

Da sich bei einer Auswertung der Settings nach MZP die Möglichkeit einer statistischen Analyse noch weiter reduzieren würde, sollen im Folgenden im Sinne einer Fallstudie einige Durchschnittswerte der selbstinitiierten Beiträge pro Minute in sechs ausgewählten Settings zu den beiden Messzeitpunkten diskutiert werden: 
Tab. 4: Anzahl und Anteil selbstinitiierter Äußerungen pro Kind in ausgewählten Sprachfördersettings

\section{selbstinitiierte \\ Äußerungen}

\begin{tabular}{|c|c|c|c|c|}
\hline & $\begin{array}{l}\text { Pro Minute } \\
\text { (gewichtet) }\end{array}$ & $\begin{array}{c}\text { Anteil an Äußerungen } \\
\text { der Kinder in \% }\end{array}$ & $\begin{array}{c}\text { Dauer des } \\
\text { Settings in Min }\end{array}$ & $\begin{array}{l}\text { Zahl der Kinder } \\
\text { im Setting* }\end{array}$ \\
\hline \multicolumn{5}{|l|}{ MZP1 } \\
\hline \multicolumn{5}{|l|}{ Additive Settings } \\
\hline $\begin{array}{l}1.1 \text { (literacy } \\
\text { Förderung) }\end{array}$ & 0.34 & 16 & $43: 00$ & $5(2)$ \\
\hline $\begin{array}{l}1.2 \text { (Erzählen, } \\
\text { Bilderbuch) }\end{array}$ & 0.50 & 25 & $41: 00$ & $5(2)$ \\
\hline $\begin{array}{l}1.3 \text { (Sprachspiel: } \\
\text { Freunde) }\end{array}$ & 0.65 & 10 & $30: 00$ & $9(4)$ \\
\hline \multicolumn{5}{|l|}{$\begin{array}{l}\text { Alltagsintegrierte } \\
\text { Settings }\end{array}$} \\
\hline $\begin{array}{l}2.1 \text { (literacy } \\
\text { Förderung) }\end{array}$ & 0.89 & 59 & $35: 00$ & $2(2)$ \\
\hline $\begin{array}{l}2.2 \text { (Werken, } \\
\text { Malen) }\end{array}$ & 1.48 & 66 & $62: 00$ & $3(3)$ \\
\hline 2.3 (Kamishibai) & 0.49 & 27 & $20: 00$ & $9(5)$ \\
\hline \multicolumn{5}{|l|}{ MZP2 } \\
\hline \multicolumn{5}{|l|}{ Additive Settings } \\
\hline $\begin{array}{l}1.1 \text { (literacy } \\
\text { Förderung) }\end{array}$ & 1.34 & 41 & $55: 00$ & $5(2)$ \\
\hline $\begin{array}{l}1.2 \text { (Erzählen, } \\
\text { Bilderbuch) }\end{array}$ & 0.53 & 18 & $54: 00$ & $5(2)$ \\
\hline $\begin{array}{l}1.3 \text { (Wortschatz: } \\
\text { Wiese) }\end{array}$ & 0.60 & 28 & 44:00 & $9(4)$ \\
\hline \multicolumn{5}{|l|}{$\begin{array}{l}\text { Alltagsintegrierte } \\
\text { Settings }\end{array}$} \\
\hline $\begin{array}{l}2.1 \text { (literacy } \\
\text { Förderung) }\end{array}$ & 1.64 & 59 & $19: 00$ & $2(2)$ \\
\hline $\begin{array}{l}2.2 \text { (Werken, } \\
\text { Malen) }\end{array}$ & 1.81 & 61 & $38: 00$ & $4(3)$ \\
\hline 2.3 (Experiment) & 0.34 & 38 & $50: 00$ & $13(6)$ \\
\hline
\end{tabular}

MZP = Messzeitpunkt. * Die Werte in Klammern geben die Zahl der Fallkinder an, deren Äußerungen in die Analyse der Selbstinitiierung eingingen. 
Die Daten der 19 Kinder zeigen große Unterschiede innerhalb der jeweiligen Gruppe der Settings. In zwei additiven Settings ist die Anzahl selbstinitiierter Äußerungen zu beiden MZP relativ niedrig, während sie im dritten zu MZP2 deutlich zunimmt. In zwei alltagsintegrierten Settings ist die Anzahl selbstinitiierter Äußerungen schon zu MZP1 deutlich höher und steigert sich auch zu MZP2 weiter. Das dritte alltagsintegrierte Setting weist dagegen eine konstant niedrige Selbstinitiierung auf und verhält sich damit wie zwei der additiven Settings.

Interessant sind genau nun die Settings, die der gängigen Erwartung: additiv = stärkere Lenkung, damit weniger Selbstinitiierung; alltagsintegriert = größere Möglichkeiten der Selbstinitiierung widersprechen, also die Settings 1.1. und 2.3. Legt man ein cut-off-Point von einer selbstinitiierten Äußerung pro Kind pro Minute fest, ergibt sich, dass keineswegs alle alltagsintegrierten Settings dieser offeneren Gesprächsstruktur entsprechen: Setting 2.3 hat in beiden, Setting 2.1 zumindest zum ersten Messzeitpunkt ebenso niedrige Werte wie die Mehrheit der additiven Settings, während das additive Setting 1.1. zumindest zu MZP2 deutlich darüber liegt.

Die hier präsentierten Ergebnisse aus dem SPRÜNGE-Projekt sind aufgrund der geringen Stichprobengröße nicht belastbar; weitere Untersuchungen stehen daher an. Sie weisen aber auf Unterschiede in der Beteiligung von Kindern unterschiedlich hoher Sprachkompetenz an Fördersituationen hin, die weder direkt von der Form des Settings (additiv oder alltagsintegriert, vgl. Tab. 4) noch vom Sprachförderbedarf der Kinder (vgl. Tab. 2) abhängig sind. Wie die Korrelationsanalyse (Tab. 1) gezeigt hat, steht die Selbstinitiierung dabei in einem mittelstarken $\mathrm{Zu}$ sammenhang zur syntaktischen Komplexität, was darauf hindeutet, dass bei einer größeren Zahl selbstinitiierter auch mehr satzförmige Äußerungen auftreten. Dies müssen keineswegs dieselben Äußerungen sein, vielmehr deutet eine höhere Selbstinitiierung auch auf eine insgesamt höhere Beteiligung hin.

\section{Bewertung/Reflexion des Instruments}

Die Forschung zu Sprachbildung und -förderung konnte in den letzten Jahren zeigen, dass es bei der Wirksamkeit von Sprachförderung weniger auf die Struktur eines Sprachförderprogramms als auf die Sprachförderstrategien der Fach- und Lehrkräfte ankommt (Beckerle, 2017; Kammermeyer et al., 2019; Kucharz, 2018). Insbesondere zeigte sich eine große Bedeutung des korrektiven Feedbacks und der semantischen oder syntaktischen Expansion. Beides sind Strategien, die keine direkten Zugzwänge für weitere Kinderäußerungen darstellen, aber sehr wohl als Anschlussstellen für selbstinitiierte Kinderäußerungen dienen (z.B. die semantische Expansion der Fachkraft in Transkript VII im Online-Anhang). Um- 
gekehrt schließen korrektive Feedbacks und semantische oder syntaktische Expansionen an Kinderäußerungen an, die ebenfalls selbstinitiiert sein können, also z.B. Fragen, Beobachtungen, Kommentare oder andere Impulse darstellen. Wenn in der Literatur mehrfach, wenn auch nicht systematisch, auf die Bedeutung kindlicher Initiative für das Zustandekommen lernförderlicher Situationen hingewiesen wird (Isler et al., 2014; Kappauf, 2018; Löffler \& Vogt, 2015; Siraj-Blatchford, 2009) zeigt dies ein Desiderat für weitere Forschung zum Zustandekommen von Kinderäußerungen. Auch bekannte bzw. aktuelle Sprachförderansätze wie das Hanen-Programm (Girolametto, Weitzmann \& Greenberg, 2003) oder die marte meo-Methode (Bünder, Sirringhaus-Bünder \& Helfer, 2018) weisen den kindlichen Initiativen und der dafür notwendigen Zurückhaltung des Erwachsenen einen hohen Stellenwert bei der Kommunikationsgestaltung zu.

Das hier vorgestellte Kategoriensystem stellt einen ersten Versuch dar, die Einbettungsform von Kinderbeiträgen in Sprachfördersettings systematisch zu bestimmen. Der Indikator ,Selbstinitiierung' erfasst für einzelne Kinder, aber auch für Settings, wie stark Kinder Gelegenheiten zum Sprechen nutzen bzw. wieviel Raum einem Kind zur Entfaltung spontaner Gesprächsbeiträge gegeben wird. An Daten von 30 Kindern zeigte sich, dass mehr Gelegenheiten zum spontanen Sprechen auch mit mehr syntaktisch komplexen Redebeiträgen von Kindern einhergehen. Dass der Anteil selbstinitiierter Äußerungen von Kindern in alltagsintegrierten Settings zwar höher, aber statistisch nicht signifikant ist, spricht unseres Erachtens gegen eine vorschnelle höhere Bewertung solcher Settings. Vielmehr zeigen die Fallstudien in beiden Arten von Sprachförderung Settings mit höheren und niedrigeren Anteilen an selbstinitiierten Beiträgen.

Für die Praxis folgt aus dieser Studie, dass Fachkräfte bei der Gestaltung von Fördersituationen dazu ermutigt werden sollten, sich stärker auf spontane Beiträge von Kindern einzulassen und nicht nur ihre eigene Agenda zu verfolgen. Bilderbuchsituationen, aber auch gemeinsames Basteln oder Werken mit nebenher laufenden informellen Gesprächen bieten häufig mehr Gelegenheiten für Kinder, ihre eigenen Vorstellungen und Erfahrungen einzubringen, als stark gelenkte Sprachspiele. Das im Projekt entwickelte Videofeedback erlaubt es, in direktem Austausch mit der Fachkraft solche Situationen im Video oder Transkript aufzusuchen und die Handlungsmöglichkeiten der Fachkraft zu erörtern. Dazu bilden die im Projekt vorgenommenen Interaktionsanalysen eine gute Grundlage.

Im Forschungskontext könnte das Instrument auch dazu dienen, qualitativ erhobene Daten leichter mit quantitativen zu verknüpfen. Interaktionsanalysen müssen sich auf eine relativ kleine Auswahl relativ kurzer Handlungssequenzen beschränken. Die dabei herausgearbeiteten Besonderheiten eines Settings lassen sich nicht verallgemeinern. Mithilfe der geringeren Zahl der Kategorien der Einbettungsanalyse wird es möglich, auch über längere Situationen hinweg und für 
eine größere Zahl an Kindern Grade der Selbstinitiierung zu bestimmen, die dann mit weiteren Daten dieser Kinder oder der Fachkräfte verknüpft werden können. Es wäre interessant, für ein größeres Sample die Zusammenhänge von selbstinitiierten Äußerungen in Sprachfördersituationen und der Entwicklung ihrer Sprachkompetenzen zu untersuchen. Auch dürfte eine genauere Analyse der Einbettungsform der Äußerungen im Abgleich mit ihren syntaktischen Strukturen neue Erkenntnisse über sprachförderliche Interaktionsformate liefern.

\section{Literatur}

Beckerle, C. (2017). Alltagsintegrierte Sprachförderung im Kindergarten und in der Grundschule. Evaluation des „Fellbach-Projekts". Weinheim: Beltz Juventa.

Becker-Mrotzek, M. \& Vogt, R. (2009). Unterrichtskommunikation. Linguistische Analysemethoden und Forschungsergebnisse (Germanistische Arbeitshefte, 2. Aufl.). Tübingen: Niemeyer.

Bergmann, J. \& Quasthoff, U. (2010). Interaktive Verfahren der Wissensgenerierung: Methodische Problemfelder. In U. Dausendschön-Gay, C. Domke \& S. Ohlhus (Hrsg.), Wissen in (Inter)Aktion. Verfahren der Wissensgenerierung in unterschiedlichen Praxisfeldern (S. 21-34). Berlin: De Gruyter.

Bruner, J. (1987). Wie das Kind sprechen lernt. Bern: Verlag Hans Huber.

Bünder, P., Sirringhaus-Bünder, A. \& Helfer, A. (Hrsg.) (2018). Lehrbuch der MarteMeo-Methode. Entwicklungsförderung mit Videounterstützung (4. Aufl.). Göttingen: Vandenhoeck und Ruprecht.

Chafe, W. (1994). Discourse, Consciousness and Time. The Flow and Displacement of Conscious Experience in Speaking and Writing. Chicago: University Chicago of Press.

Egert, F., Quehenberger, J., Dederer, V. \& Wirts, C. (2018). Kindliche Initiative als Qualitätsindikator bei sprachlichen Bildungsaktivitäten. Ergebnisse einer empirischen Untersuchung. Diskurs Kindheits- und Jugendforschung, 4, 489-494.

Ehlich, K., Bredel, U. \& Reich, H. H. (2008). Sprachaneignung - Prozesse und Modelle. In Bundesministerium für Bildung und Forschung (Hrsg.), Referenzrahmen zur altersspezifischen Sprachaneignung (S. 9-34). Bonn: BMBF.

Ennemoser, M., Kuhl, J. \& Pepouna, S. (2013). Evaluation des Dialogischen Lesens zur Sprachförderung bei Kindern mit Migrationshintergrund. Zeitschrift für Pädagogische Psychologie, 27(4), 229-239.

Erdogan, E., Betz, T., Kämpfe, K., Kucharz, D., Mehlem, U. \& Rezagholinia, S. (im Druck). Dimensionen der Sprachförderung am Übergang Kindergarten-Grundschule. In S. Gentrup, S. Henschel, K. Schotte, L. Beck, \& P. Stanat, (Hrsg.), Sprach- und Schriftsprachförderung gestalten: Evaluation von Qualität und Wirksamkeit umgesetzter Konzepte. Stuttgart: Kohlhammer.

Flick, U. (2019). Gütekriterien qualitativer Sozialforschung. In N. Baur \& J. Blasius (Hrsg.), Handbuch Methoden der empirischen Sozialforschung (S. 473-488). Wiesbaden: VS.

F4transkript Version 5.40.0. (2015). Benutzerhandbuch f4transkript. Verfügbar unter: https:// www.hwg-lu.de/fileadmin/user_upload/service/it-service-center/anleitungen/f4_manual_de.pdf [02.09.2019].

Garfinkel, H. (1967). Studies in Ethnomethodology. Prentice Hall: Englewood Cliffs/N.J.

Girolametto, L., Weitzman, E., \& Greenberg, J. (2003). Training day care staff to facilitate children's language. American Journal of Speech-Language Pathology, 12, 299-311.

Goodwin, C. (1981). Conversational organization: Interaction between speakers and hearers. New York: Academic Press.

Hagstedt, H. \& Krauth, I. M. (2014). Mit Lernwerkstätten Schule entwickeln. In H. Hagstedt \& I. M. Krauth (Hrsg.), Lernwerkstätten, Potenziale für Schulen von morgen (S. 8-19). Frankfurt (Main): Grundschulverband. 
Hausendorf, H. \& Quasthoff, U. (2005). Sprachentwicklung und Interaktion. Eine linguistische Studie zum Erwerb von Diskursfähigkeiten. Radolf: Verlag für Gesprächsforschung.

Heller, V. (2012). Kommunikative Erfahrungen von Kindern in Familie und Unterricht: Passungen und Divergenzen. Tübingen: Stauffenburg.

Hormann, O. \& Koch, K. (2014). Von Strukturen zu Strategien und Interaktionen Sprachförderung am Übergang in die Grundschule. Befunde aus dem Projekt EvaniK. In A. Wegner \& E. Vetter (Hrsg.), Mehrsprachigkeit und Professionalisierung in pädagogischen Berufen. Interdisziplinäre Zugänge zu aktuellen Herausforderungen im Bildungsbereich (S. 77-93). Opladen: Barbara Budrich.

Isler, D., Künzli, S. \& Wiesner, E. (2014). Alltagsgespräche im Kindergarten - Gelegenheitsstrukturen für den Erwerb bildungssprachlicher Fähigkeiten. Schweizerische Zeitschrift für Bildungswissenschaften, 36(3), 459-479.

Kammermeyer, G., King, S., Goebel, P., Lämmerhirt, A., Leber, A., Metz, A., Papillion-Piller, A. \& Roux, S. (2019). Mit Kindern im Gespräch - Qualifizierungskonzept zur Sprachbildung und Sprachförderung von Kindern in Kindertageseinrichtungen. In C. Titz, S. Geyer, A. Ropeter, H. Wagner, S. Weber \& M. Hasselhorn (Hrsg.), Konzepte zur Sprach- und Schriftsprachförderung: Praxiserfahrungen (S. 13-36). Stuttgart: Kohlhammer.

Kannengieser, S. \& Tovote, K. (2015). Alltagsintegrierte Sprachförderung in der Spielgruppe - Welche Fachperson-Kind-Interaktionen finden statt? Schweizerische Zeitschrift für Bildungswissenschaften, 37(1), 57-74.

Kappauf, N. (2018). Interaktionsverhalten von frühpädagogischen Fachkräften: Qualitätsentwicklung durch online-basiertes Videofeedback. Dissertation, LMU München.

Kieferle, C., Reichert-Garschhammer, E. \& Becker-Stoll, F. (2013). Sprachliche Bildung von Anfang an. Strategien, Konzepte und Erfahrungen. Göttingen: Vandenhoeck \& Ruprecht.

Knapp, W., Ricart Brede, J., Gasteiger-Klicpera, B. \& Kucharz, D. (2009). Sprachliche Förderung von Kindern im Vorschulalter - Eine exemplarische Videoanalyse. In: K. Schramm \& C. Schroeder (Hrsg.), Empirische Zugänge zu Sprachförderung und Spracherwerb in Deutsch als Zweitsprache (S.157-184). Münster: Waxmann.

König, A. (2009). Interaktionsprozesse zwischen ErzieherInnen und Kindern. Eine Videostudie aus dem Kindergartenalltag. Wiesbaden: VS.

Kucharz, D. (2018). Qualifizierung der Fachkräfte im Elementarbereich. In C. Titz, S. Geyer, A. Ropeter, H. Wagner, S. Weber \& M. Hasselhorn (Hrsg.), Konzepte zur Sprach- und Schriftsprachförderung entwickeln (S. 249-261). Stuttgart: Kohlhammer.

Kucharz, D., Mehlem, U., Rezagholinia, S. \& Erdogan, E. (2018). Sprachförderung und sprachliche Entwicklung ein- und mehrsprachiger Kinder im letzten Kindergartenjahr. Empirische Pädagogik, 32(2), 177-197.

Kuckartz, U. (2010). Einführung in die computergestützte Analyse qualitativer Daten (3. Aufl.). Wiesbaden: VS.

Löffler, C. \& Vogt, F. (2015). Strategien der Sprachförderung im Kita-Alltag. München: Ernst Reinhardt Verlag.

Lüders, M. (2003). Unterricht als Sprachspiel: eine systematische und empirische Studie zum Unterrichtsbegriff und zur Unterrichtssprache. Bad Heilbrunn: Klinkhardt.

Maas, U. (2010). Orat und literat. Grundbegriffe der Analyse gesprochener und geschriebener Sprache. Grazer Linguistische Studien, 73, 21-150.

Mehan, H. (1979). What time is it, Denise? Asking known information questions in classroom discourse. Theory into Practice, 28(4), 285-294.

Melhuish, E. (2013). Research on Early Childhood Education in the United Kingdom. In M. Stamm \& D. Edelmann (Hrsg.), Handbuch frühkindliche Bildungsforschung (S. 211-221). Wiesbaden: VS.

Meyer-Drawe, K. (1984). Der fruchtbare Moment im Bildungsprozess. Zu Copeis phänomenologischem Ansatz pädagogischer Theoriebildung. In H. Danner \& W. Lippitz (Hrsg.), Beschreiben 
- Verstehen - Handeln. Phänomenologische Forschungen in der Pädagogik (S. 91-151). München: Röttger.

Morek, M. (2012). Kinder erklären. Interaktionen in Familie und Unterricht im Vergleich. Tübingen: Stauffenburg.

Petermann, F. (2010). Sprachstandserhebungstest für Fünf- bis Zehnjährige (SET 5-10). München: Hogrefe. Quasthoff, U. (2012). Aktual- und mikrogenetische Zugänge zur Ontogenese: Inspirationen der Konversationsanalyse zur Verbindung von sprachlichen Praktiken und dem Erwerb sprachlicher Kompetenzen. In R. Ayaß \& C. Meyer (Hrsg.), Sozialität in Slow Motion: Theoretische und empirische Perspektiven (S. 217-244). Wiesbaden: VS.

Rehbein, J. (2007). Erzählen in zwei Sprachen - auf Anforderung. In K. Meng \& J. Rehbein (Hrsg.). Kindliche Kommunikation - einsprachig und mehrsprachig (S. 392-460). Münster: Waxmann.

Sacks, H., Schegloff, E. A. \& Jefferson, G. (1974). A simplest systematics for the organization of turntaking for conversation. Language, 50, 696-735.

Schegloff, E. (1996). Turn Organization: One Intersection of Grammar and Interaction, In E. Ochs, E. A. Schegloff \& S. Thompson (eds.), Interaction and Grammar (pp. 52-133). Cambridge: Cambridge University Press.

Schmidt, T. (2016). EXMARaLDA Partitur-Editor Manual Version 1.6. Verfügbar unter: https://www. exmaralda.org/pdf/Partitur-Editor_Manual.pdf [02.09.2019].

Selting, M., Auer, P., Barden, B., Bergmann, J., Couper-Kuhlen, E., Günthner, S., Meier, C., Quasthoff, U., Schlobinski, P. \& Uhmann, S. (1998). Gesprächsanalytisches Transkriptionssystem (GAT). Linguistische Berichte, 173, 91-122.

Siraj-Blatchford, I. (2009). Conceptualising progression in the pedagogy of play and sustained shared thinking in early childhood education: a Vygotskian perspective. Education and Child Psychology, 26(2), 77-89.

Ulich, M. \& Mayr, T. (2003). Sismik. Sprachverhalten und Interesse an Sprache bei Migrantenkindern in Kindertageseinrichtungen (Beobachtungsbogen und Begleitheft). Freiburg: Herder.

Ulich, M. \& Mayr, T. (2006). Seldak. Sprachentwicklung und Literacy bei deutschsprachig aufwachsenden Kindern (Beobachtungsbogen und Begleitheft). Freiburg: Herder.

\section{Autor*innen}

Univ.-Prof. Dr. Ulrich Mehlem

Goethe-Universität Frankfurt a. M./Institut für Pädagogik der Elementar- und Primarstufe

Theodor-W.-Adorno-Platz 6, 60323 Frankfurt am Main

mehlem@em.uni-frankfurt.de

Ezgi Erdogan

Goethe-Universität Frankfurt a. M./Institut für Pädagogik der Elementar- und Primarstufe

Theodor-W.-Adorno-Platz 6, 60323 Frankfurt am Main

erdogan@em.uni-frankfurt.de

Dieser Artikel ist inklusive Anhang abrufbar unter:

https://doi.org/10.35468/5801_07 


\section{Online Anhang}

Dieses Herausgeberwerk und alle vorgestellten Instrumente zur Erfassung institutioneller (schrift-)sprachlicher Bildung sind online verfügbar unter: https://doi.org/10.35468/5801 


\section{Codebuch für die Erfassung der Einbettung und der syntaktischen Struktur sprachlicher Beiträge von Kindern in Sprachförderaktivitäten. Online-Material zur Vertiefung}

Ulrich Mehlem \& Ezgi Erdogan

Zitiervorschlag:

Mehlem, U. \& Erdogan, E. (2019). Codebuch für die Erfassung der Einbettung und der syntaktischen Struktur sprachlicher Beiträge von Kindern in Sprachförderaktivitäten. Online-Material zur Vertiefung. In K. Mackowiak, C. Beckerle, S. Gentrup \& C. Titz (Hrsg.), Instrumente zur Erfassung institutioneller (schrift-)sprachlicher Bildung (Online-Anhang). Bad Heilbrunn: Klinkhardt.

Verfügbar unter: https://doi.org/10.35468/5801_07 


\section{Vorbemerkungen}

Das Kategoriensystem eignet sich für die Analyse von transkribierten Videoaufnahmen in vorschulischen Settings. Es setzt ein detailliertes Transkript der videografierten Szene mit EXMARALDA (Schmidt, 2016) oder F4 (F4transkript, 2015) voraus, das sich möglichst an den Konventionen von GAT orientieren sollte (Selting et al., 1998). In einem solchen Transkript können verbale und relevante nonverbale Beiträge einer Gruppeninteraktion in einer Partiturschreibweise (wie in EXMARALDA, vgl. die Beispiele in Abschnitt 6) oder sequentiell in Zeilen angeordnet sein. Wichtig ist, dass die sprachlichen Überlappungen und parallele Handlungen auch grafisch, z.B. durch eckige Klammern, anzeigt werden. Die Weiterverarbeitung der Daten kann dann mit MAXQDA (Kuckartz, 2010; MAXQDA, 2018) erfolgen, möglich ist aber auch die Verwendung von EXCEL. Zur Erfassung der Einbettung der sprachlichen Beiträge der Kinder ist es wichtig, dass der Kontext den analysierenden Forschenden bekannt ist, zumindest eine der auswertenden Personen sollte bei der Sprachfördersituation anwesend sein und unmittelbar im Anschluss ethnographische Notizen angefertigt haben. Bei der Durchführung der Einbettungsanalyse sollte neben dem Transkript auch das Videomaterial selbst zur Verfügung stehen, um nonverbale Interaktionsanteile zu überprüfen.

Auf der Grundlage dieser in elektronischer Form vorliegenden Daten können weitere quantitative Analysen durchgeführt werden, wie sie ansatzweise im Buchbeitrag ausgeführt wurden.

\section{Bestimmung der Kodiereinheiten der Einbettungsanalyse}

Um bei der Analyse sprachlicher Interaktionen zu einer Bestimmung aussagekräftiger Kodiereinheiten zu gelangen, müssen immer drei Ebenen unterschieden werden:

1. Ebene der Interaktion zwischen verschiedenen Sprecher*innen, die individuelle Beiträge zur gemeinsamen sprachlichen Interaktion leisten. Das grundlegende Prinzip der Aufteilung der Gesprächsanteile ist der Sprecher*innenwechsel. Die von eine*r Sprecher*in in einem Interaktionsbeitrag getätigte Äußerung ist ein Turn (Schegloff, 1996).

2. Ebene der sprachlichen Struktur (Grammatik): Die größte grammatische Einheit ist der Satz, der aus mehreren Teilsätzen bestehen kann. Unterhalb des Satzes sind aber auch kleinere grammatische Einheiten als sprachliches Material eines Turns möglich, über Wortgruppen und Phrasen bis hin zu einzelnen Wörtern oder Partikeln. Lässt sich ein Turn in mehrere konstituierende Einheiten (Turn Constituting Units, TUC) gliedern, muss für jede von ihnen die syntaktische Struktur bestimmt werden. Ein vollständiger Satz liegt vor, wenn zu einem verbalen Prädikat alle obligatorischen Argumentpositionen besetzt sind. (Schegloff, 1996)

3. Ebene der Prosodie: Im Anschluss an Selting (1995) und Selting et al. (1998) wird Prosodie verstanden „,... als Oberbegriff für diejenigen suprasegmentalen Aspekte der Rede, die sich aus dem Zusammenspiel der akustischen Parameter Grundfrequenz (F0), Intensität und Dauer in silbengroßen oder größeren Domänen ergeben. Hierzu gehören auditive Phänomene wie Intonation, d.h. der Tonhöhenverlauf gesprochener Sprache in der Zeit, Lautstärke, Länge, Pause, sowie die damit zusammenhängenden komplexeren Phänomene Sprechgeschwindigkeit/Tempo und Rhythmus" (Selting, 1995, S. 1). Die größte prosodische Einheit ist die Phrasierungseinheit (bzw. Intonationseinheit), der ein Intonationsverlauf entspricht. In Transkripten wird die Tonhöhenbewegung am Ende der Phrasierungseinheit markiert. Einer fallenden Intonation entspricht im Deutschen in der Regel eine Aussage, einer steigenden eine Frage. In dieser Funktion werden daher auch die gängigen Interpunktionszeichen verwendet. Jede Phrasierungseinheit weist einen Primärakzent, also eine mit höherer Lautstärke gesprochene Silbe auf, die durch Großschreibung markiert wird. Die Phrasierungseinheit kann mit einer grammatischen Einheit zusammenfallen, sie aber auch überschreiten.

Für die Analyse der Einbettungsform der Interaktionsbeiträge eines Kindes ist die relevante Kodiereinheit der Turn im Sinne von (1). Eine Äußerung wird auch dann als einziger Turn kodiert, wenn sie durch Interjektionen unterbrochen wird oder mit anderen Äußerungen überlappt, solange kein definitiver Sprecher*innenwechsel eintritt. Ein Turn kann aus mehreren Konstituenten (Turn Constituting Units, TCU) z.B. in Form von Phrasierungs- oder grammatischen Einheiten bestehen. Diese werden für die Einbettungsanalyse nicht getrennt berücksichtigt, da sie jeweils als Ganzes den Gesprächsbeitrag ausmachen (Schegloff, 1996). 
Über diese Klassifizierung alltäglicher Konversationen hinaus stehen bei der Sprachförderung größere Diskurseinheiten (Hausendorf \& Quasthoff, 2005) im Fokus, in denen ein*e primäre*r Sprecher*in in einem Turn über mehrere Sätze/Phrasierungseinheiten hinweg einen komplexeren Sachverhalt darstellt. Im Sinne einer Förderung der diskursiven Sprachkompetenz kommt es darauf an, dass die Fachkräfte Kinder dabei unterstützen, solche sprachlichen Einheiten zu produzieren. Die Analyse der Einbettungsformen kindlicher Äußerungen muss daher diesen Aspekt besonders berücksichtigen. Bei solchen satzübergreifenden Diskurseinheiten eines einzelnen Kindes wird jede weitere satzförmige kindliche Äußerung genauso wie die Äußerung kategorisiert, mit der der kindliche Redebeitrag eröffnet wurde. Für die syntaktische Analyse müssen dagegen alle einen Turn konstituierenden Einheiten jeweils für sich betrachtet und analysiert werden.

Die folgenden Tabellen unterscheiden die hier beschriebenen Fälle und geben jeweils Ankerbeispiele aus dem Korpus des Projekts SPRÜNGE ${ }^{1}$, die in Abschnitt 6 dokumentiert werden. Um die Orientierung zu erleichtern, wird mit der römischen Ziffer jeweils die Nummer des Settings, mit arabischen die Nummer des Einzelsegments (graue Kopfzeile im Transkriptblock vor der Zeitangabe in eckigen Klammern) angegeben. Die Nummerierung der Transkriptblöcke (linke Ziffer in eckigen Klammern) ist für das Auffinden der Äußerungen nicht relevant.

Die Beschreibung des Codes gibt die notwendigen Bedingungen an, die für die Vergabe des Codes erfüllt sein müssen; ggf. werden unterschiedliche Bedingungen angegeben. Für manche der Ankerbeispiele werden kurze eigene Beschreibungen gegeben, die die allgemeine Beschreibung des Codes spezifizieren. In den Ankerbeispielen werden meistens Auszüge aus den Transkripten und die dort übliche Transkription (z.B. Großschreibung bei Akzent) verwendet. Die Schriftart wurde vereinheitlicht.

\section{Regeln für die Abgrenzung von Äußerungen und die Bestimmung von Kodiereinheiten}

\begin{tabular}{|l|l|l|l|}
\hline Oberkategorie & Bezeichnung des Codes & $\begin{array}{l}\text { Beschreibung des } \\
\text { Codes }\end{array}$ & Ankerbeispiel(e) \\
\hline $\begin{array}{l}\text { Eigenständige } \\
\text { Äußerung }\end{array}$ & $\begin{array}{l}\text { Einzeläußerung nach und } \\
\text { vor Sprecher*innen- } \\
\text { wechsel (turn by turn- } \\
\text { talk) }\end{array}$ & $\begin{array}{l}\text { Das Kind spricht zu } \\
\text { Ende, bevor ein Spre- } \\
\text { cher*innenwechsel } \\
\text { erfolgt. Der Turn } \\
\text { entspricht einer } \\
\text { Phrasierungseinheit. }\end{array}$ & $\begin{array}{l}\text { FK schaut auf Buch und dann } \\
\text { wieder in Sitzkreis } \\
\text { K1: man kann man auch sie } \\
\text { ZWICKN } \\
\text { FK: (1) kann man sich auch } \\
\text { ZWICKN gä } \\
\text { (I/55-57) }\end{array}$ \\
\hline $\begin{array}{l}\text { Eigenständige } \\
\text { Äußerung }\end{array}$ & $\begin{array}{l}\text { Eigenständige Äußerung } \\
\text { ohne Sprecher*innen- } \\
\text { wechsel in } \\
\text { satzübergreifender } \\
\text { Diskurseinheit }\end{array}$ & $\begin{array}{l}\text { Satzübergreifende Dis- } \\
\text { kurseinheit. Jeder neue } \\
\text { Satz bzw. jede neue } \\
\text { Phrasierungseinheit zählt } \\
\text { als eigene Äußerung. }\end{array}$ & $\begin{array}{l}\text { K1: ehm meine MAma:: hat } \\
\text { geSAHAGT (--) weil (--) wir } \\
\text { GEHN heut in den URlaub } \\
\text { (IV/17) }\end{array}$ \\
\hline $\begin{array}{l}\text { Zusammenfassung } \\
\text { kleinerer nicht } \\
\text { eigenständiger } \\
\text { Äußerungen }\end{array}$ & $\begin{array}{l}\text { Fragmentierte } \\
\text { Einzeläußerungen, } \\
\text { Sprecher*innenwechsel / }\end{array}$ & $\begin{array}{l}\text { Mehrere Wörter / Phra- } \\
\text { sierungseinheiten zählen } \\
\text { als eine Äußerung, } \\
\text { obwohl sie durch } \\
\text { Inberlappung }\end{array}$ & $\begin{array}{l}\text { FK: (legt zwei Äpfel auf den } \\
\text { Tisch und fordert Kinder auf, } \\
\text { die Frucht zu benennen)K1: } \\
\text { [KIRSCH] [apfl] FK: ja K1: } \\
\text { grüner APfel (III/259-263) }\end{array}$ \\
\hline
\end{tabular}

\section{Die Codes der Einbettungsanalyse von Äußerungen}

Im Zentrum des Analyseinstruments stehen Kategorien zur Beschreibung der Einbettungsform der kindlichen Interaktionsbeiträge in das laufende Interaktionsgeschehen. Es geht hier darum, den Äußerungskontext vor dem relevanten Turn des Kindes daraufhin zu untersuchen, inwieweit dieser durch einen Zugzwang einer*s anderen Interaktionsteilnehmers*in in hohem Maße erwartbar ist oder nicht. Unter dem Begriff Zugzwang werden Merkmale von Äußerungen oder anderen interaktiven Zügen der anderen Gesprächsteilnehmer*innen zusammengefasst, die den folgenden Gesprächsbeitrag als besonders wahrscheinlich bzw. sein Nicht-Eintreten selbst als erklärungsbedürftig erscheinen lassen (Heller, 2012; Morek, 2012). Hierzu zählen Fragen oder Aufforderungen, die entweder direkt oder indirekt an das im Anschluss sprechende Kind gerichtet sind.

\footnotetext{
${ }^{1}$ Weitere Hinweise zum Projekt SPRÜNGE finden Sie in dem Buchbeitrag und in anderen Projektpublikationen (Kucharz, Mehlem, Rezagholinia \& Erdogan, 2018; Erdogan, Betz, Kämpfe, Kucharz, Mehlem \& Rezagholinia, o.J.).

${ }^{2}$ In diesem Beispiel bilden zwei Äußerungen des Kindes zusammen einen Turn. Erst danach tritt Sprecher*innenwechsel ein.

${ }^{3} \mathrm{~K} 1$ repariert seine erste Äußerung [kirsch] unmittelbar im Anschluss [apfl] und elaboriert sie dann weiter [grüner apfl]. 
Zugzwänge können lokaler und globaler Natur sein. Bei globalen Zugzwängen wird erwartet, dass der Angesprochene die Rolle eines*r primären Sprechers*in in einer satzübergreifenden Diskurseinheit übernimmt. Lokale Zugzwänge beziehen sich nur auf eine unmittelbare folgende Äußerung. Zugzwänge können direkt oder indirekt an das im Anschluss sprechende Kind gerichtet sein. Bei direkten Zugzwängen wird das Kind z.B. durch Namensnennung direkt adressiert. In (V/44) verbindet die Lehrkraft ihre Frage mit der Namensnennung, nachdem zuvor ein anderes Kind geantwortet hat, obwohl K2 mit der Aktivität an der Reihe war.

Ein Zugzwang kann in der unmittelbar vorausgehenden Äußerung der Fachkraft oder eines anderen Kindes enthalten sein wie in (V/44) oder etwas weiter zurückliegen. Bei der Bilderbuchbetrachtung bedient der Beitrag von K1 (I/52) einen Zugzwang der Fachkraft, auf den schon ein anderes Kind geantwortet hat. Gleichzeitig lässt sich dieser Beitrag als Anschluss an dessen Äußerung beschreiben, allerdings ohne dass ein Zugzwang von Seiten des Kindes K4 vorliegt.

Da es einfacher ist, im vorgängigen Interaktionsgeschehen Zugzwänge zu erkennen als lose Anschlüsse, wird nach dem Ausschlussprinzip verfahren. Selbstinitiierte Beiträge bilden also zunächst eine Restkategorie, für die sich im unmittelbaren oder mittelbaren Vorfeld kein Zugzwang finden ließ. In der bereits beschriebenen Bilderbuchszene (I) ist das zum Beispiel der Fall bei der Äußerung von K1 (I/56). Diese Äußerung ist ein weiterer Kommentar zu der gerade im Fokus befindlichen Bilderbuchszene. Die unmittelbar vorausgehende Handlung der Fachkraft (I/55) stellt keinen Zugzwang dar, schließt aber die Interaktion zu der Bilderbuchseite auch nicht dadurch ab, dass sie weiterliest. Sie bereitet einen weiteren Kommentar zu der Szene dadurch vor, dass sie vom Buch nochmals in die Runde schaut. Diese Vorgehensweise würde bei der gängigen Zählung von Sprachförderstrategien nicht berücksichtigt, obwohl sie einen interaktiven Beitrag zur Äußerung von K1 (I/56) leistet.

Im Fokus der Einbettungsanalyse stehen ausschließlich sprachliche Äußerungen von Kindern. Auch wenn nonverbale Äußerungen die Funktion von Turns erfüllen können und tatsächlich einen wichtigen Beitrag für den Vollzug der Interaktion leisten, werden sie im Folgenden nur berücksichtigt, wenn sie mit parallelen bzw. folgenden sprachlichen Äußerungen im Zusammenhang stehen.

Eine weitere Abgrenzung ist nötig gegenüber Äußerungen, die unverständlich sind oder nicht im engeren Sinne aus Worten bestehen. Diskurspartikeln oder Interjektionen, die eine Vielzahl kommunikativer Funktionen erfüllen, aber nicht im engeren Sinn Wörter sind, werden daher ebenfalls nicht berücksichtigt.

In der Kodierungstabelle werden zunächst die Oberkategorien aufgelistet, die für die quantitative Auswertung relevant sind. Es folgen jeweils Unterkategorien, mit denen unterschiedliche Gesprächskonstellationen unterschieden werden.

\begin{tabular}{|c|c|c|c|c|}
\hline & Oberkategorie & Bezeichnung des Codes & $\begin{array}{l}\text { Beschreibung des } \\
\text { Codes }\end{array}$ & Ankerbeispiel(e) \\
\hline \multirow[t]{2}{*}{1} & \multirow{2}{*}{$\begin{array}{l}\text { Vollzug eines indirekten } \\
\text { Zugzwangs der Fachkraft } \\
\text { (FK) } \\
\text { IZFK }\end{array}$} & \multirow[t]{2}{*}{$\begin{array}{l}\text { Antwort auf nicht direkt } \\
\text { an das Kind gerichtete } \\
\text { Frage der FK }\end{array}$} & \multirow{2}{*}{$\begin{array}{l}\text { Die Frage der FK } \\
\text { richtet sich an alle } \\
\text { Kinder bzw. ist nicht } \\
\text { direkt an das Kind } \\
\text { gerichtet, das } \\
\text { antwortet. }\end{array}$} & $\begin{array}{l}\text { FK: [WIE] hört sich } \\
\text { das an? - K3: Wasser } \\
(\mathrm{V} / 35)\end{array}$ \\
\hline & & & & $\begin{array}{l}\text { FK: wie SCHAUen } \\
\text { denn die geSICHter } \\
\text { aus von den BÄRN? } \\
\text { K4: böse (I/48-49) }\end{array}$ \\
\hline $2 a$ & $\begin{array}{l}\text { Vollzug eines direkten } \\
\text { Zugzwangs der FK (mit } \\
\text { Rederechtserteilung) } \\
\text { DZFK }\end{array}$ & $\begin{array}{l}\text { Antwort auf direkt an } \\
\text { Kind gerichtete Frage } \\
\text { der FK }\end{array}$ & $\begin{array}{l}\text { Die Frage der FK } \\
\text { richtet sich direkt an } \\
\text { das Kind, das } \\
\text { antwortet. Dies kann } \\
\text { durch } \\
\text { Namensnennung, } \\
\text { Blickkontakt oder } \\
\text { Zeigegeste der FK } \\
\text { ausgedrückt werden. }\end{array}$ & $\begin{array}{l}\text { FK: (Name K2) was } \\
\text { meinst DU denn? } \\
\text { K2: der lacht nich } \\
\text { (I/81) }\end{array}$ \\
\hline $2 b$ & $\begin{array}{l}\text { Vollzug eines direkten } \\
\text { Zugzwangs der } \\
\text { FK(Aufforderung) } \\
\text { DZFK }\end{array}$ & $\begin{array}{l}\text { Das Kind übernimmt } \\
\text { eine Aufgabe, z.B. in } \\
\text { einem Spiel und } \\
\text { begleitet diese } \\
\text { sprachlich. }\end{array}$ & $\begin{array}{l}\text { Die Aufforderung der } \\
\text { FK unterstützt die } \\
\text { Routine des } \\
\text { Spielverlaufs. }\end{array}$ & $\begin{array}{l}\text { FK: Jetzt darfst DU } \\
\text { einen freund aufdecken } \\
\text { K5 (beugt sich vor und } \\
\text { greift nach Karte und }\end{array}$ \\
\hline
\end{tabular}




\begin{tabular}{|c|c|c|c|c|}
\hline & & & & $\begin{array}{l}\text { schaut sie an): ein } \\
\text { Pferd (II/283) }\end{array}$ \\
\hline $3 a$ & $\begin{array}{l}\text { Anschluss an die } \\
\text { Äußerung eines anderen } \\
\text { Kindes ZKi }\end{array}$ & $\begin{array}{l}\text { Das Kind schließt an } \\
\text { Äußerung eines anderen } \\
\text { Kindes an. }\end{array}$ & $\begin{array}{l}\text { Ein Kind nimmt } \\
\text { Bezug auf die } \\
\text { Äußerung eines } \\
\text { anderen Kindes. }\end{array}$ & $\begin{array}{l}\text { K1: du hast mich } \\
\text { ANgemalt. K2: hab ich } \\
\text { nicht (VI/256-258 }\end{array}$ \\
\hline $3 b$ & $\begin{array}{l}\text { Anschluss an die } \\
\text { Äußerung eines anderen } \\
\text { Kindes } \mathrm{ZKi}\end{array}$ & $\begin{array}{l}\text { Antwort auf Frage eines } \\
\text { Kindes }\end{array}$ & $\begin{array}{l}\text { Ein Kind antwortet } \\
\text { auf die Frage eines } \\
\text { anderen Kindes. }\end{array}$ & $\begin{array}{l}\text { K3: echte? } \\
\text { K2: [es gibt auch] } \\
\text { Babyhaie (VI/554) }\end{array}$ \\
\hline $4 \mathrm{a}$ & $\begin{array}{l}\text { Selbstinitiierter Beitrag } \\
\text { SI }\end{array}$ & Frage (an FK oder Kind) & $\begin{array}{l}\text { Das Kind stellt eine } \\
\text { Frage zu einem vorher } \\
\text { thematisierten oder } \\
\text { neuen Sachverhalt. }\end{array}$ & $\begin{array}{l}\text { K3: DARF ich mal } \\
\text { se:hen den meeres- } \\
\text { schmetterling (VI/545) }\end{array}$ \\
\hline $4 \mathrm{~b}$ & $\begin{array}{l}\text { Selbstinitiierter Beitrag } \\
\text { SI }\end{array}$ & $\begin{array}{l}\text { Themenfortführung, } \\
\text { Kommentar }\end{array}$ & $\begin{array}{l}\text { Das Kind äußert sich } \\
\text { zu einem vorher } \\
\text { thematisierten } \\
\text { Sachverhalt. }\end{array}$ & $\begin{array}{l}\text { FK schaut auf Buch } \\
\text { und dann wieder in } \\
\text { Sitzkreis } \\
\text { K1: kann man auch sie } \\
\text { ZWICKN (I/44-45) }\end{array}$ \\
\hline $4 c$ & $\begin{array}{l}\text { Selbstinitiierter Beitrag } \\
\text { SI }\end{array}$ & $\begin{array}{l}\text { Thematisierung eines } \\
\text { neuen Sachverhalts }\end{array}$ & $\begin{array}{l}\text { Das Kind äußert sich } \\
\text { zu einem neuen } \\
\text { Sachverhalt } \\
\text { (Themenwechsel). }\end{array}$ & $\begin{array}{l}\text { K4: der junge mit des } \\
\text { blauen jacke is meine } \\
\text { FREUND }(\mathrm{V} / 602)^{4}\end{array}$ \\
\hline $4 d$ & $\begin{array}{l}\text { Selbstinitiierter Beitrag } \\
\text { SI }\end{array}$ & $\begin{array}{l}\text { Handlungsbegleitendes } \\
\text { Sprechen }\end{array}$ & $\begin{array}{l}\text { Das Kind begleitet } \\
\text { eine eigene Handlung } \\
\text { sprachlich. }\end{array}$ & $\begin{array}{l}\text { K1: ich NEHme sogar } \\
\text { ein meeres- } \\
\text { schmetterling (VI/543) }\end{array}$ \\
\hline \multirow[t]{2}{*}{$4 \mathrm{e}$} & \multirow[t]{2}{*}{$\begin{array}{l}\text { Selbstinitiierter Beitrag } \\
\text { SI }\end{array}$} & \multirow[t]{2}{*}{$\begin{array}{l}\text { Das Kind äußert einen } \\
\text { Wunsch. }\end{array}$} & \multirow[t]{2}{*}{$\begin{array}{l}\text { Das Kind äußert sich } \\
\text { zu etwas, wovon } \\
\text { gerade die Rede war } \\
\text { (Wunsch). }\end{array}$} & $\begin{array}{l}\text { FK fragt nach einem } \\
\text { Geräusch, andere } \\
\text { Kinder antworten } \\
\text { K4: DES nehm ich } \\
(\mathrm{V} / 38)\end{array}$ \\
\hline & & & & $\begin{array}{l}\text { Ein anderes Kind } \\
\text { bekommt eine Karte, } \\
\text { die es zuordnen soll. } \\
\text { K: ich auch }(\mathrm{V} / 875)\end{array}$ \\
\hline $4 \mathrm{f}$ & $\begin{array}{l}\text { Selbstinitiierter Beitrag } \\
\text { SI }\end{array}$ & $\begin{array}{l}\text { Sich melden mit } \\
\text { anschließender } \\
\text { Rederechtserteilung } \\
\text { durch FK, ohne dass die } \\
\text { Frage von ihr ausging }\end{array}$ & $\begin{array}{l}\text { Nicht nur die Melde- } \\
\text { geste oder der verbale } \\
\text { Wunsch, sondern auch } \\
\text { der folgende Beitrag } \\
\text { des Kindes wird als } \\
\text { selbstinitiiert gewertet, } \\
\text { da die Rederechts- } \\
\text { erteilung der FK vom } \\
\text { Kind ausgelöst wurde. }\end{array}$ & $\begin{array}{l}\text { K1: ich! (IV/102) } \\
\text { (reklamiert Rederecht), } \\
\text { FK stellt Beitrag } \\
\text { zurück, bis anderes } \\
\text { Kind fertig ist } \\
\text { FK: was wolltest du } \\
\text { sagen? } \\
\text { K1: ehm (2) Mama... } \\
\text { (IV/110) }\end{array}$ \\
\hline $5 \mathrm{a}$ & Mitsprechen MS & $\begin{array}{l}\text { Mitsprechen (u.U. nach } \\
\text { Aufforderung) }\end{array}$ & $\begin{array}{l}\text { Die FK und alle } \\
\text { Kinder der Gruppe } \\
\text { sprechen zusammen } \\
\text { einen Text. Das } \\
\text { Fokuskind spricht } \\
\text { gleichzeitig mit den } \\
\text { anderen. }\end{array}$ & $\begin{array}{l}\text { K1: [alle kinder sind } \\
\text { jetz fit bist du's } \\
\text { AUCH? dann MACH } \\
\text { gleich mit] (V/6-7) }\end{array}$ \\
\hline $5 b$ & Mitsingen MSG & Mitsingen bei Lied & $\begin{array}{l}\text { Die FK leitet das } \\
\text { gemeinsame Singen } \\
\text { ein. }\end{array}$ & $\begin{array}{l}\text { K5: [bunte gruppe } \\
\text { bunte gruppe] (II/139) }\end{array}$ \\
\hline $5 c$ & Nachsprechen NS & $\begin{array}{l}\text { Nachsprechen nach } \\
\text { Aufforderung }\end{array}$ & $\begin{array}{l}\text { Die FK fordert dazu } \\
\text { auf, ein Wort oder } \\
\text { einen Satz } \\
\text { nachzusprechen. }\end{array}$ & $\begin{array}{l}\text { LK fordert zum } \\
\text { Nachsprechen auf: K4, } \\
\text { K5: [SCHÜSSEL (-) } \\
\text { TEE] (V/309-9) }\end{array}$ \\
\hline
\end{tabular}

\footnotetext{
${ }^{4}$ Während eines Spiels schaut ein Kind aus dem Fenster und sieht dort andere Kinder.
} 


\section{Codierungen der sprachlichen Form der Äußerung}

Die grammatische Form der Äußerung ist wichtig, um den tatsächlichen Umfang der sprachlichen Beteiligung eines Kindes zu erfassen. Sie bildet die Grundlage für die Bestimmung der durchschnittlichen Äußerungslänge. In dieser Analyse wird vorgeschlagen, für die sprachliche Komplexität nur die vollständigen Sätze pro Minute zu berücksichtigen. Entscheidend ist also nicht nur Überschreitung der Grenze der Einwortäußerung. Die Frage der grammatischen Richtigkeit spielt dagegen bei der Kodierung der Einbettungsform (vgl. 3) keine Rolle. Syntaktische Vollständigkeit ist nur bei der Kategorie ,Satz' notwendig.

\begin{tabular}{|l|l|l|}
\hline Bezeichnung des Codes & Beschreibung des Codes & Ankerbeispiel \\
\hline $\begin{array}{l}\text { Einzelwortäußerung } \\
\text { (Diskurspartikeln) }\end{array}$ & $\begin{array}{l}\text { Es handelt sich zwar um Wörter, } \\
\text { deren Bedeutung aber nur im Kontext } \\
\text { klar wird (Synsemantika). }\end{array}$ & K4: NEIN (I/78) \\
\hline Einzelwortäußerung (Inhaltswörter) & $\begin{array}{l}\text { Das Wort hat einen eigenständigen } \\
\text { semantischen Kern (Autosemantika). }\end{array}$ & K4: böse (I/49) \\
\hline Erweiterte Nominalphrase & $\begin{array}{l}\text { Ein um mindestens ein Element } \\
\text { erweitertes Nomen (meist } \\
\text { Determinierer oder Adjektiv) } \\
\text { vorhanden. }\end{array}$ & K1: grüner Apfel (III/263) \\
\hline Wortgruppe & $\begin{array}{l}\text { Mehrere Wörter, die keine Phrase } \\
\text { bilden. }\end{array}$ & K1: roter und grün (III/265) \\
\hline Anvollständige satzförmige & $\begin{array}{l}\text { Die Äußerung enthält ein Verb, ohne } \\
\text { dass alle obligatorischen } \\
\text { Argumentpositionen besetzt sind. }\end{array}$ & $\begin{array}{l}\text { K4: ich schau auch die } \\
\text { (III/178) [es fehlt die } \\
\text { Verbartikel AN oder die } \\
\text { Präposition AUF)] }\end{array}$ \\
\hline Vollständige satzförmige Äußerung & $\begin{array}{l}\text { Die obligatorischen Argument- } \\
\text { positionen eines Verbs sind besetzt. }\end{array}$ & $\begin{array}{l}\text { K1: zwei Augen sind zu } \\
\text { (I/52) }\end{array}$ \\
\hline Unverständliche Äußerungen & $\begin{array}{l}\text { Nicht oder nicht vollständig } \\
\text { verständlich, kommunikative } \\
\text { Funktion sind nicht oder nur teilweise } \\
\text { erschließbar. }\end{array}$ & $\begin{array}{l}\text { Bei der Äußrung von K1 in } \\
\text { VI/521 ist weder die } \\
\text { kommunikative Funktion } \\
\text { noch die grammatische } \\
\text { Struktur erschließbar. }\end{array}$ \\
\hline Keine wortförmigen Äußerungen & $\begin{array}{l}\text { Zustimmung, Verneinung oder } \\
\text { emotionale Markierung wird } \\
\text { signalisiert. }\end{array}$ & $\begin{array}{l}\text { FK: [bah:] (Die FK drückt } \\
\text { die Emotion ,böse aus, } \\
\text { I/50) }\end{array}$ \\
\hline
\end{tabular}

\section{Umgang mit Zweifelsfällen}

- Kommunikationsbeiträge zwischen Kindern (auch Streit etc.) werden als „Anschluss an Kind“ kodiert, wenn sie deutlich als Reaktion auf die Äußerung eines anderen Kindes erkennbar sind.

- Äußerungen, in denen ein Kind die Interaktion mit einem anderen Kind eröffnet, werden als „selbstinitiiert“ kodiert.

- Tierstimmen etc. (Grunzen o.ä.) sind als verbale Äußerungen zu werten, auch wenn sie in der nonverbalen Spur transkribiert werden. Sie zählen aber nicht als wortförmige Äußerungen und werden nicht kodiert.

- Nonverbale Anteile (z.B. Meldegesten) oder Lachen werden nicht kodiert, aber für die Kodierung der nächsten verbalen Äußerung berücksichtigt.

\section{Transkriptauszüge}

Die folgenden Transkriptauszüge dienen der genaueren Überprüfung der in den Tabellen genannten Ankerbeispiele. Die im Zuge der Einbettungsanalyse vergebenen Codes werden in einer Spalte links vom Zeilenkopf des Transkripts eingetragen. Die Transkripte wurden mit dem Programm EXMARALDA angefertigt und entsprechen einer Partiturschreibweise. Neben den verbalen Äußerungen, jeweils dem Namen des Sprechers mit dem Kürzel [v] zugeordnet, gibt es auch eine nonverbale Spur, in der für das Verständnis der verbalen Interaktion relevante Handlungen festgehalten sind. Diese werden mit dem Kürzel [nv] nach dem Namen am Zeilenkopf markiert. 


\begin{tabular}{|c|c|c|}
\hline CODE & {$[41]$} & 49 [24:31.9] \\
\hline IZFK & $\begin{array}{l}\text { FK }[v] \\
\text { FK }[n v] \\
\text { K4 }[\mathbf{v}] \\
\text { K2 }[n v]\end{array}$ & wie SCHAUEN denn die GESICHTER aus von den BÄRN? \\
\hline & [42] & $51[24: 35.0] \quad 52[24: 35.8]$ \\
\hline $\begin{array}{l}\text { IZFK } \\
\text { IZFK }\end{array}$ & $\begin{array}{l}\text { FK }[\mathbf{v}] \\
\text { FK }[\mathrm{nv}] \\
\text { K1 [v] } \\
\text { K4 [v] } \\
\text { K4 [nv] }\end{array}$ & $\begin{array}{l}\text { [bah:] } \begin{array}{l}\text { joa: } \\
\text { nickt leichtbei "oah } \\
\text { [ZWEI }\end{array} \\
\text { [ein auge] is ZU: } \\
\text { bei "zu" schlägt einmal mit der Faust in die Luft }\end{array}$ \\
\hline & [43] & $53[24: 40.3]$ \\
\hline Ftsg & $\begin{array}{l}\text { FK }[\mathbf{v}] \\
\text { FK }[\mathrm{nv}] \\
\text { K1 }[\mathbf{v}] \\
\text { K2 }[\mathrm{nv}]\end{array}$ & $\begin{array}{l}\text { schauen die] ganz OAH ich halt die rosa FEST(-)joa } \\
\text { ich halt die" streckt Arm in die Luft } \\
\begin{array}{ll}\text { augen] sind zu } & \text { beginnt sich zu strecken }\end{array}\end{array}$ \\
\hline & [44] & $56[24: 45.9]$ \\
\hline SI & $\begin{array}{l}\text { FK }[\mathbf{v}] \\
\text { FK }[\mathbf{n v}] \\
\text { K1 [v] }\end{array}$ & $\begin{array}{l}\text { (1) genau } \\
\text { schaut auf Buch und dann wieder in Sitzkreis }\end{array}$ \\
\hline & [45] & $57[24: 47.9]$ \\
\hline Ftsg & $\begin{array}{l}\text { FK }[\mathbf{v}] \\
\text { FK }[\mathbf{n v}] \\
\text { K1 [v] }\end{array}$ & $\begin{array}{l}\text { (1) kann man sich auch ZWICKN gä schaut ma } \\
\text { kann man auch sie ZWICKN }\end{array}$ \\
\hline
\end{tabular}

Spätere Szene:

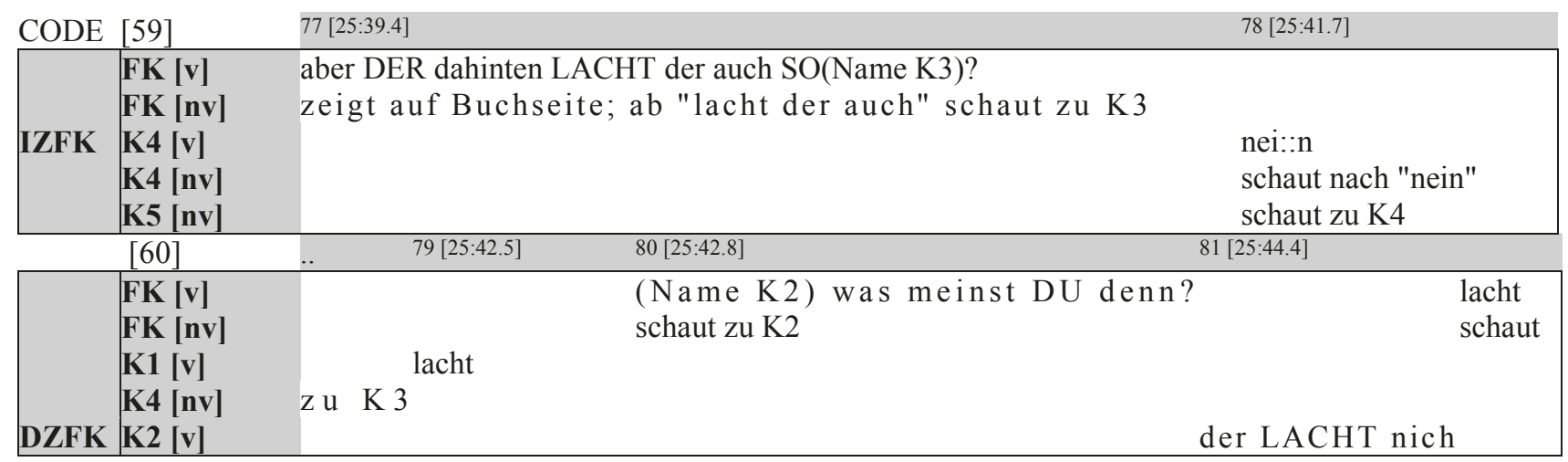

II. Kartenspiel

[175] 282 [14:52.1]

F [v] und jetzt jetz machen wir es im UHRZEIGERSINN (-) jetzt ist die [176]

F [v] G(Name K5) dran denn die UHR geht immer so rum jetzt darfst DU einen freund F [nv] zeigt auf K5; zeigt bei "geht immer" einmal im Uhrzeigersinn den Kreis entlang

[177]

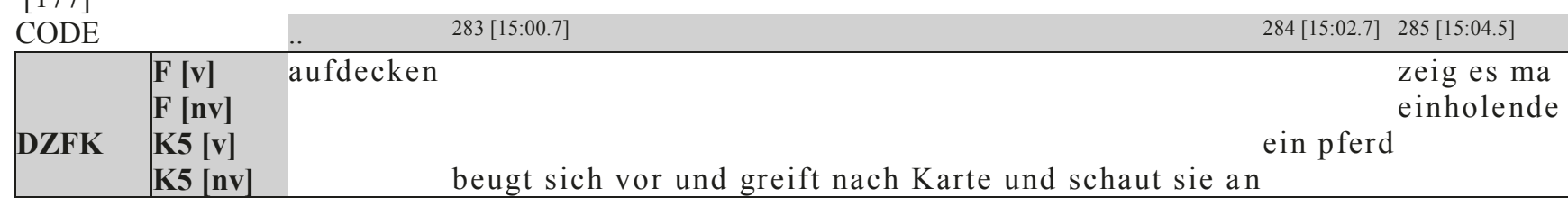




\begin{tabular}{|ll}
\hline $\mathbf{F}[\mathbf{v}]$ & allen ob es ein pferd ist \\
$\mathbf{F}[\mathbf{n v}]$ & Handbewegung; beugt sich leicht vor \\
$\mathbf{K 5}[\mathbf{n v}]$ & dreht Karte in Richtung vom Sitzkreis \\
\hline
\end{tabular}

III. Wortschatzarbeit mit Obst

\begin{tabular}{|c|c|c|}
\hline CODE & [168] & $261[12: 12.2] 262[12: 13.1] 263[12: 13.7]$ \\
\hline IZFK & $\begin{array}{l}\text { F [ [v] } \\
\text { F [nv] } \\
\text { K3 [v] } \\
\text { K4 [v] }\end{array}$ & 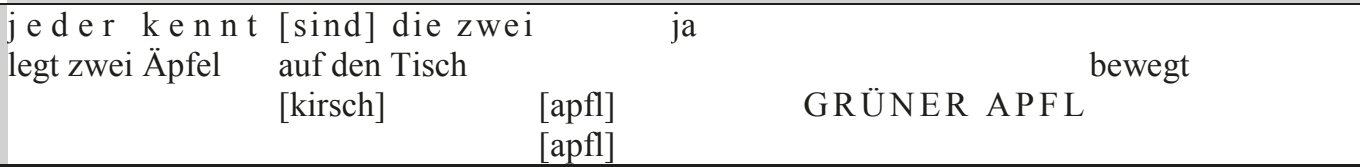 \\
\hline & {$[169]$} & $267[12: 17.1]$ \\
\hline IZFK & $\begin{array}{l}\mathbf{F}[\mathbf{v}] \\
\mathbf{F}[\mathbf{n v}] \\
\mathbf{K} 3[\mathbf{v}]\end{array}$ & $\begin{array}{llll}\text { Oberkörper zu K3 } & \text { ein GRÜNA apfl } & \text { EIN ROTER } \\
\text { roter und grün } & \text { ROTER apfl } & \end{array}$ \\
\hline & {$[170]$} & $269[12: 19.3]$ \\
\hline SI & $\begin{array}{l}\mathrm{F}[\mathbf{v}] \\
\mathrm{K} 2[\mathbf{v}] \\
\mathrm{K} 2[\mathbf{n v}]\end{array}$ & 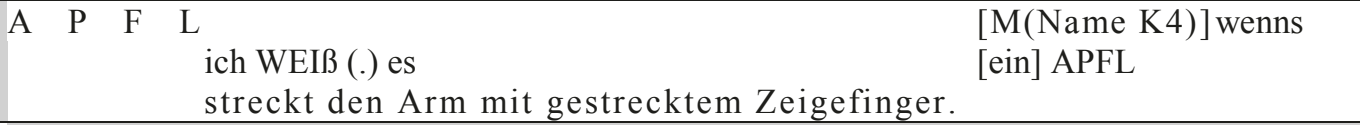 \\
\hline & {$[171]$} & $272[12: 24.2] \quad 273[12: 25.2] 274[12: 25.8]$ \\
\hline $\begin{array}{l}\text { IZFK } \\
\text { SI } \\
\text { DZFK }\end{array}$ & \begin{tabular}{|l|}
$\mathbf{F}[\mathbf{v}]$ \\
$\mathrm{K} 3[\mathbf{v}]$ \\
$\mathrm{K} 1[\mathbf{v}]$ \\
$\mathrm{K} 4[\mathbf{v}]$ \\
\end{tabular} & $\begin{array}{l}\text { [DIE] APFL } \\
\text { Ä:::PFEL [aus } \\
{[\text { die }]} \\
\text { [das }\end{array}$ \\
\hline & {$[172]$} & $277[12: 31.6]$ \\
\hline & \begin{tabular}{|l} 
F [v] \\
K3 [v] \\
K1 [v]
\end{tabular} & $\begin{array}{l}\text { d e m A : w i r d e i n Ä ] und ich hab jetz auf meine karte geschrieben DER } \\
\text { [ÄPFEL] } \\
\text { hab ich gewusst] }\end{array}$ \\
\hline & [173] & $278[12: 36.9]$ \\
\hline SI & $\begin{array}{l}\text { F [ }[\mathbf{v}] \\
\text { K4 [v] } \\
\text { K4 [nv] }\end{array}$ & $\begin{array}{l}\text { ich schau auch die } \\
\text { beugt sich über den tisch } \\
\text { und greift nach Früchten }\end{array}$ \\
\hline
\end{tabular}

IV. Erzählung im Morgenkreis

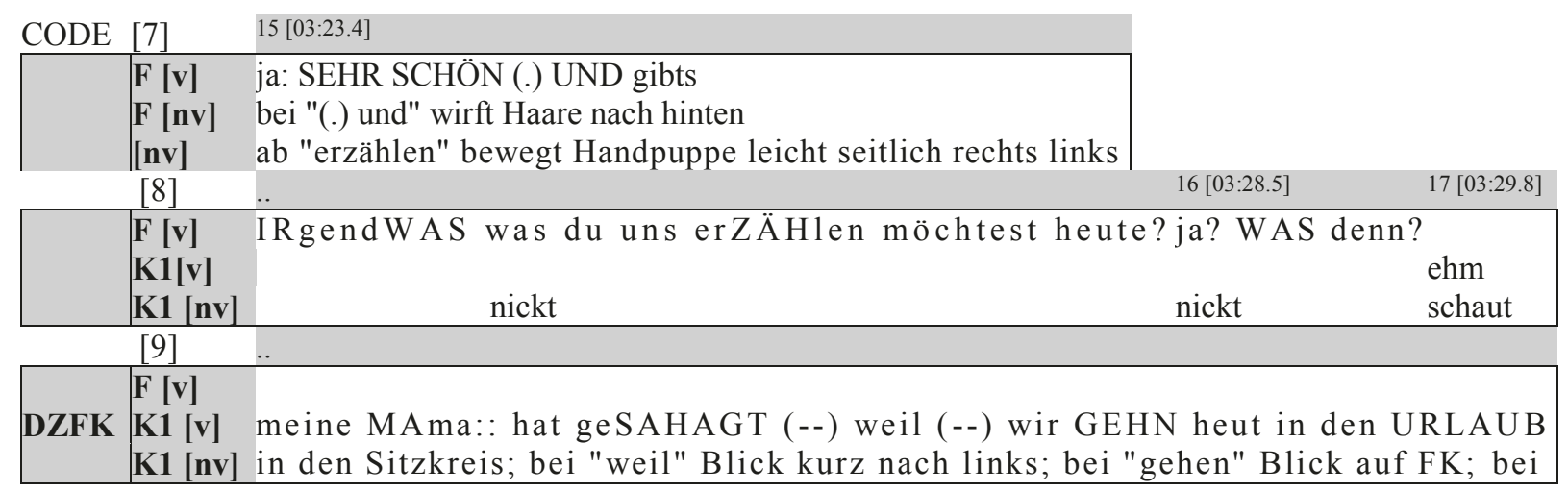




\begin{tabular}{|c|c|c|c|}
\hline & {$[10]$} & .. & $18[03: 39.6] 19[03: 40.4]$ \\
\hline \multirow{4}{*}{ DZFK } & F [v] & \multirow[b]{4}{*}{ "heut in den Urlaub" Blick abgewendet mach links } & heute? \\
\hline & F [nv] & & nickt nickt bei "Wellen (-) \\
\hline & K1 [v] & & da gibt's so GROßE \\
\hline & K1 [nv] & & s schaut in den \\
\hline & [11] & .. & $20[03: 46.1] 21[03: 46.6]$ \\
\hline & $\begin{array}{l}\text { F [v] } \\
\text { F [nv] }\end{array}$ & & $\begin{array}{l}\text { ohja } \\
\text { nickt }\end{array}$ \\
\hline & $\begin{array}{l}\text { K1 [v] } \\
\text { K1 [nv] }\end{array}$ & $\begin{array}{l}\text { WELLN (-) und da war mal ein STUAM (--) } \\
\text { S i t z k r e is ; b e i "St u a m " s c ha u t F k }\end{array}$ & $\begin{array}{l}\text { ein RIESIGN } \\
\text { schaut in den Sitzkreis }\end{array}$ \\
\hline
\end{tabular}

Spätere Szene: Nachdem andere Kinder ebenfalls im Erzählkreis dran waren, reklamiert K1 nochmals das Rederecht für sich (IV/102) und bekommt es schließlich erteilt (IV/110).

[58]

\begin{tabular}{|c|c|c|c|}
\hline \multicolumn{2}{|c|}{ CODE } & \multicolumn{2}{|c|}{$101[06: 46.5] 102[06: 46.9]$} \\
\hline \begin{tabular}{|l} 
SI \\
DZFK \\
\end{tabular} & \begin{tabular}{|l} 
F $[\mathbf{v}]$ \\
F $[\mathbf{n v}]$ \\
K1 [v] \\
K4 $[\mathbf{v}]$
\end{tabular} & SPIELEN können & $\begin{array}{l}\text { [schön wa/] WAS habt ihr denn GESPIELT? } \\
\text { ab "was" wendet sich zu K1 streichelt } \\
\text { [i/ ich] }\end{array}$ \\
\hline $\begin{array}{l}{[59]} \\
\text { CODE }\end{array}$ & & & $103[06: 49.9]$ \\
\hline DZFK & $\begin{array}{l}\text { F }[\mathbf{v}] \\
\text { F }[\mathrm{nv}] \\
\text { K4 [v] } \\
\text { K4 [nv] }\end{array}$ & $\begin{array}{l}\text { gleich (Name K1) } \\
\text { K1 über den Rücken }\end{array}$ & $\begin{array}{l}\text { richtet Blick zurück zu K4; bei "fängt" nickt } \\
\text { wir ham so ein KISSN wer als erstes des KISSN } \\
\text { Blickkontakt mit Fk; bei "Strich" malt Strich mit }\end{array}$ \\
\hline
\end{tabular}

[67]

\begin{tabular}{|c|c|c|c|}
\hline CODE & & 109 & $110[07: 26.7]$ \\
\hline & $\begin{array}{l}\mathbf{F}[\mathbf{v}] \\
\mathbf{F}[\mathbf{n v}]\end{array}$ & $\begin{array}{l}\text { (Name K1) was wolltest du grad SAGEN? } \\
\text { sich zu K1 legt kurz Hand auf den Rücken }\end{array}$ & Blick zu K1; bei \\
\hline SI & $\begin{array}{l}\text { K1 [ }[\mathbf{v}] \\
\text { K1 [nv] }\end{array}$ & & $\begin{array}{l}\text { e hm (2) mama (--) M A M A (- } \\
\text { Blick nach vorne; bei "hat }\end{array}$ \\
\hline
\end{tabular}

\section{Spiel mit Montessori-Material (Geräuschedosen, LK: Lehrkraft)}

Die Kinder setzen um einen Tisch, der mit einem grünen Tuch bedeckt ist. Immer ein Kind zieht unter dem Tuch eine Dose hervor und schüttelt sie, um ein Geräusch wahrzunehmen.

[32]

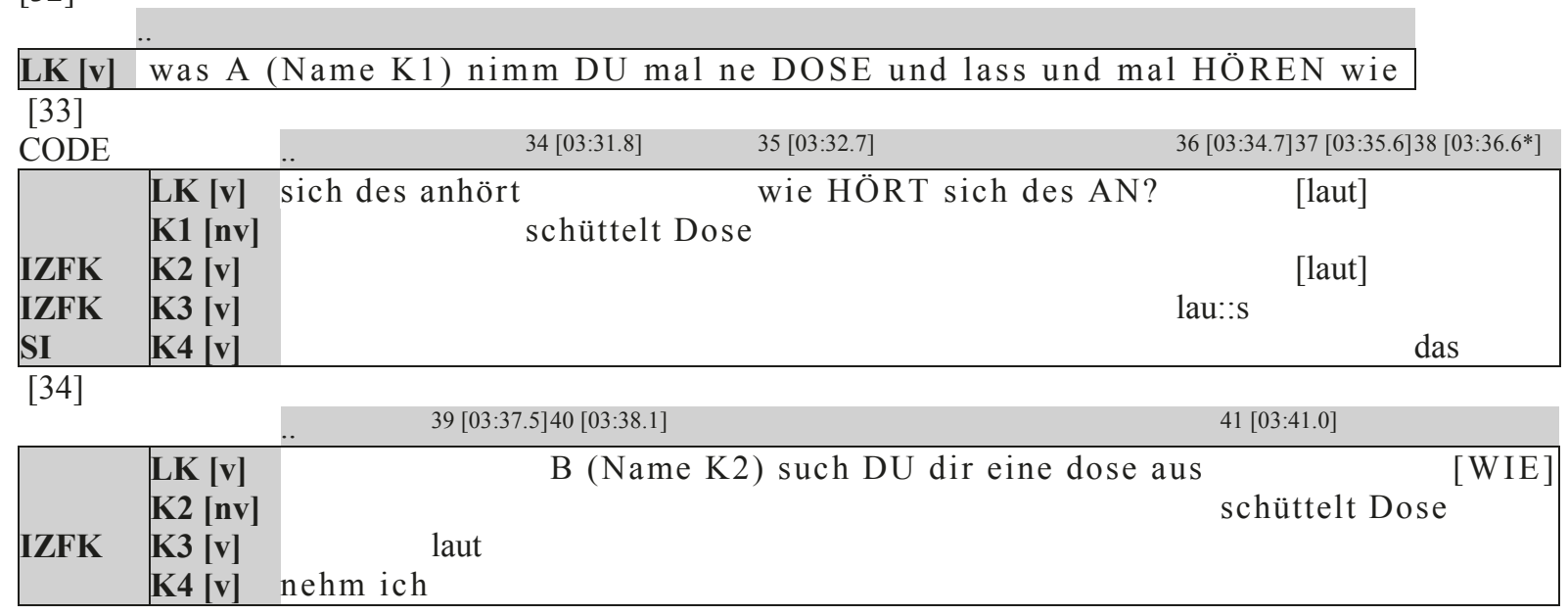




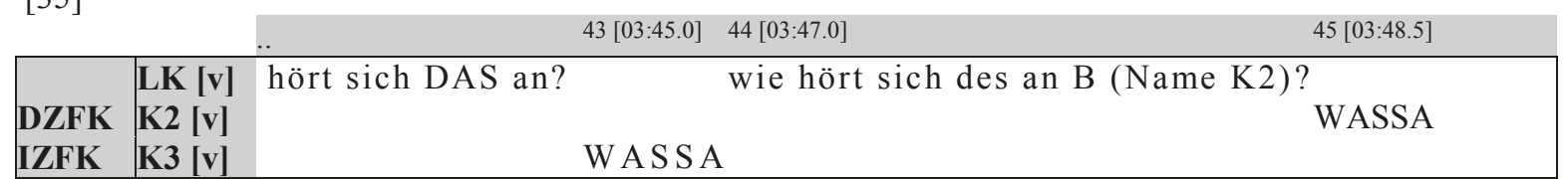

Spätere Szene: In dieser Szene gibt es Karten zu Quatschversen, in denen bestimmte Gegenstände, die nichts miteinander zu tun haben, kombiniert werden.

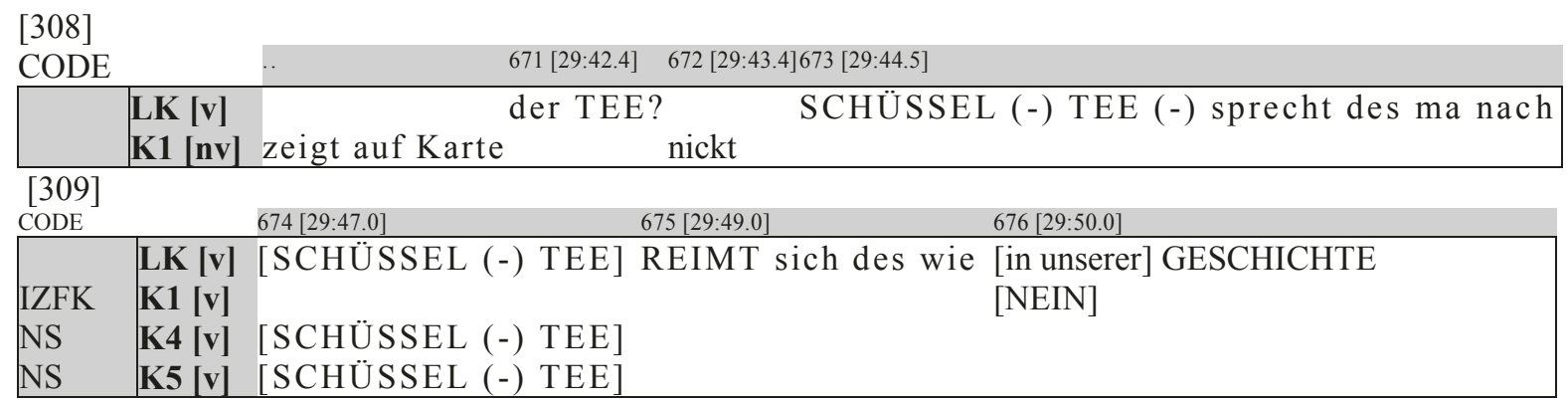

\section{Lernwerkstatt: Malen und Hämmern}

In der Szene arbeiten zwei Jungen (K1 und K3) an einem Fußballfeld aus Holz, in dessen Ränder Nägel eingeschlagen werden. Das Mädchen K2 malt ein Bild, ein weiteres Mädchen kommt später hinzu. Die Fachkraft steht bei den Kindern und schaut ihnen bei der Arbeit zu.

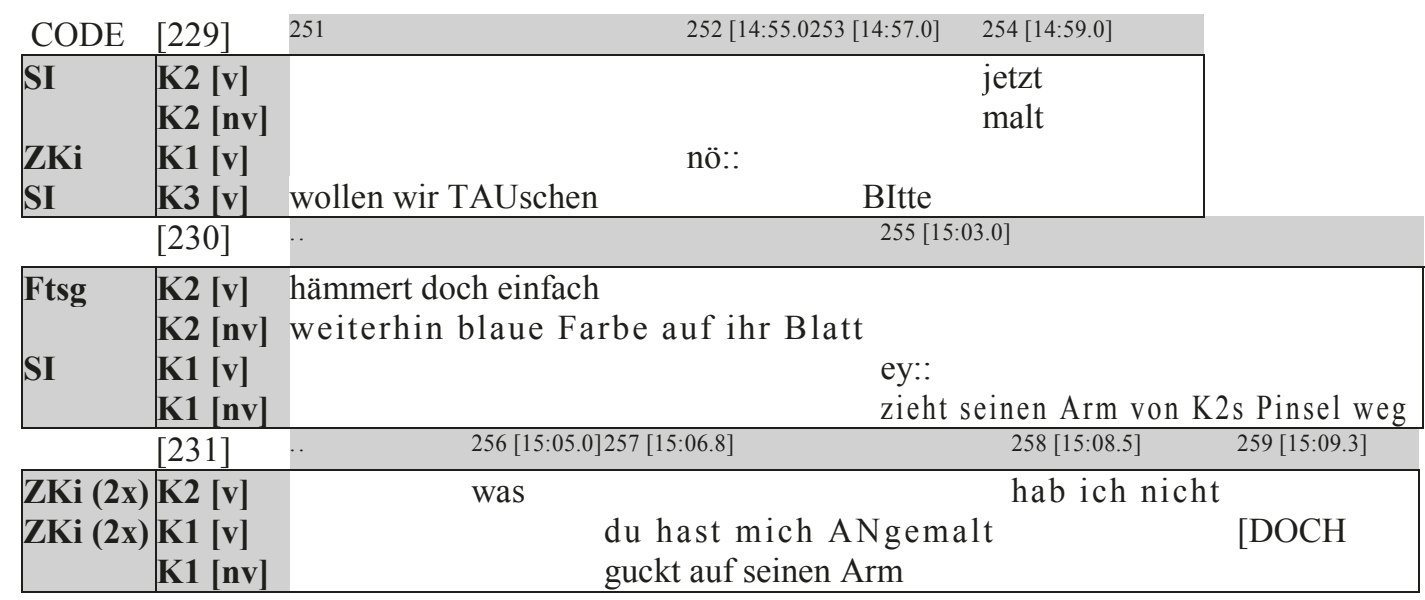

Spätere Szene

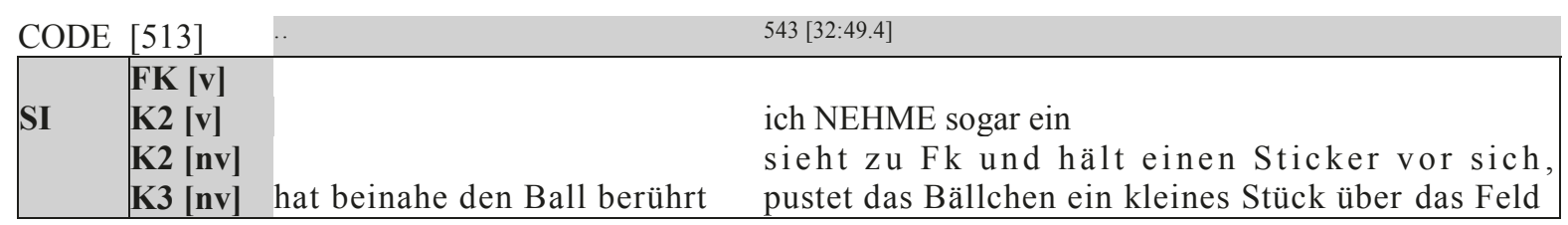


[514]

\begin{tabular}{|c|c|c|}
\hline ODE & & $544[32: 53.1]$ \\
\hline tsg & $\begin{array}{l}\text { FK }[\mathbf{v}] \\
\text { K2f }[\mathbf{v}] \\
\text { K2f }[\mathbf{n v}] \\
\text { K1 }[\mathbf{n v}]\end{array}$ & $\begin{array}{l}\text { MEERESschmetterling } \\
\text { be i " M E E RE S s c h m e t t e r l i ng " }\end{array}$ \\
\hline $\begin{array}{l}{[515]} \\
\text { CODE }\end{array}$ & & 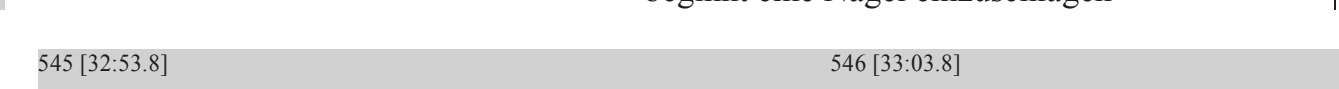 \\
\hline & $\begin{array}{l}\mathrm{K} 2[\mathbf{v}] \\
\mathrm{K} 2[\mathrm{nv}] \\
\mathrm{K} 3[\mathrm{v}] \\
\mathrm{K} 3[\mathrm{nv}]\end{array}$ & $\begin{array}{l}\text { GIBT es das e:cht (.) die tiere? } \\
\text { hält den Sticker hoch, nach "mee:resschmetterling"sieht z u Fk, be i "e::cht" } \\
\text { DARF ich mal se:hen den mee:resschmetterling (4) } \\
\text { zu K2 }\end{array}$ \\
\hline
\end{tabular}

[516]

$\begin{array}{lll}\text { CODE } & 547[33: 06.8] & 548[33: 07.6]\end{array}$

\begin{tabular}{|l|l|}
\hline \multirow{5}{*}{ FK [v] wie bitte? } & $\begin{array}{l}\text { FK }[\mathbf{n v}] \\
\text { betritt den Bildausschnitt geht neben K2 in die Hocke } \\
\text { K2 [v] } \\
\text { K2 }[\mathbf{n v}]\end{array}$ \\
\hline
\end{tabular}

[517]

CODE

\begin{tabular}{|l|lr|}
\hline & FK [v] & ja. (2) die gibts im meer und \\
SI & $\begin{array}{ll}\text { FK [nv] klebt einen Sticker auf ihr Bild } \\
\text { K4 [v] }\end{array}$ & aber keine haie. aber keine haie (unverständ1ich) \\
K4 [nv] & kommt in Raum 1 stellt sich zu FK und K2
\end{tabular}

\begin{tabular}{l|lll|}
\hline 518$]$ & & & \\
CODE & $551[33: 17.6]$ & $552[33: 18.8]$ \\
\hline SI & FK [v] & gibts \\
\hline
\end{tabular}

[519]

$\begin{array}{llrl}\text { CODE } & \text {.. } & 553[33: 21.2] & 554[33: 22.0]\end{array}$

\begin{tabular}{|l|lll|}
\hline ZKi & FK $[\mathbf{v}]$ & au:ch. auch unterschiedliche & [ja::] \\
& K2 $[\mathbf{v}]$ & & {$[$ es gibt auch] } \\
& K1 [nv] & & nimmt einen weiteren Nagel \\
SI & K4 [v] & echte? & \\
\hline
\end{tabular}

[520]

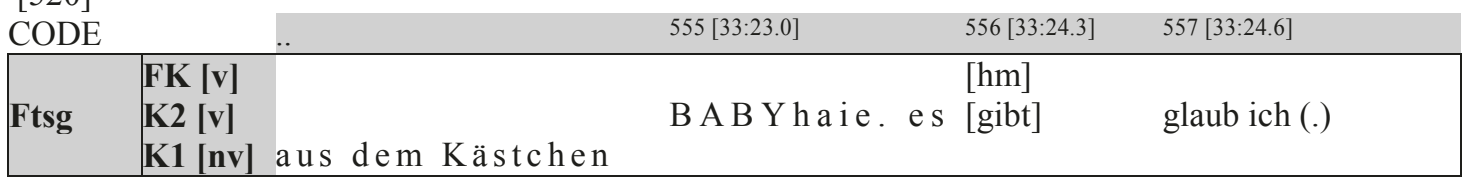

\begin{tabular}{|c|c|c|c|}
\hline [521] & & & \\
\hline CODE & & & 559 [33:27.5] \\
\hline & FK $[\mathbf{v}]$ & & [ich weiß nicht] \\
\hline SI & K2 [v] & süßhaie? gibts die? & \\
\hline & K1 [v] & & xxx xxx (unverständlich) [xxx xxx (unverständlich)] \\
\hline & K1 [nv] & & sieht zu K3 setzt seinen Nagel auf das Feld \\
\hline [522] & & & \\
\hline CODE & & 60 [33:28.1] & \\
\hline
\end{tabular}

VII. Lernwerkstatt: „Zuckerwürfel“

In der Szene haben sich die Fachkraft und sechs Kinder um einen Tisch versammelt, auf dem eine Pyramide aus Zuckerwürfeln aufgebaut ist. Es wird untersucht, was passiert, wenn die Pyramide mittels einer Pipette mit gefärbtem Wasser beträufelt wird.

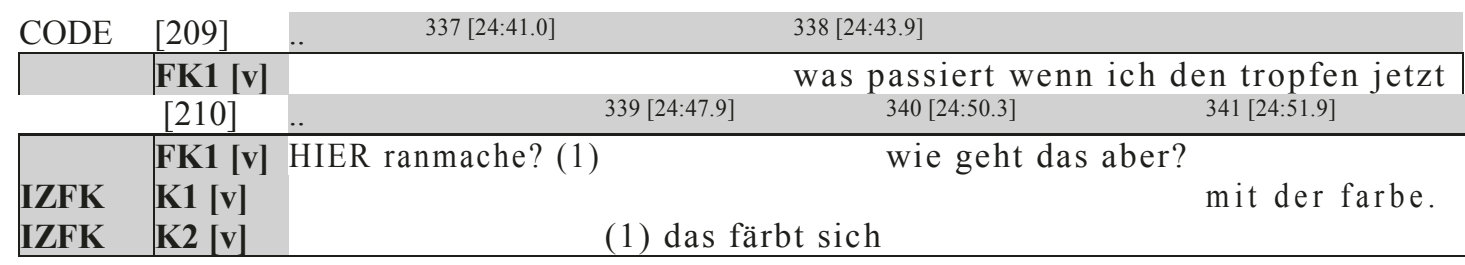




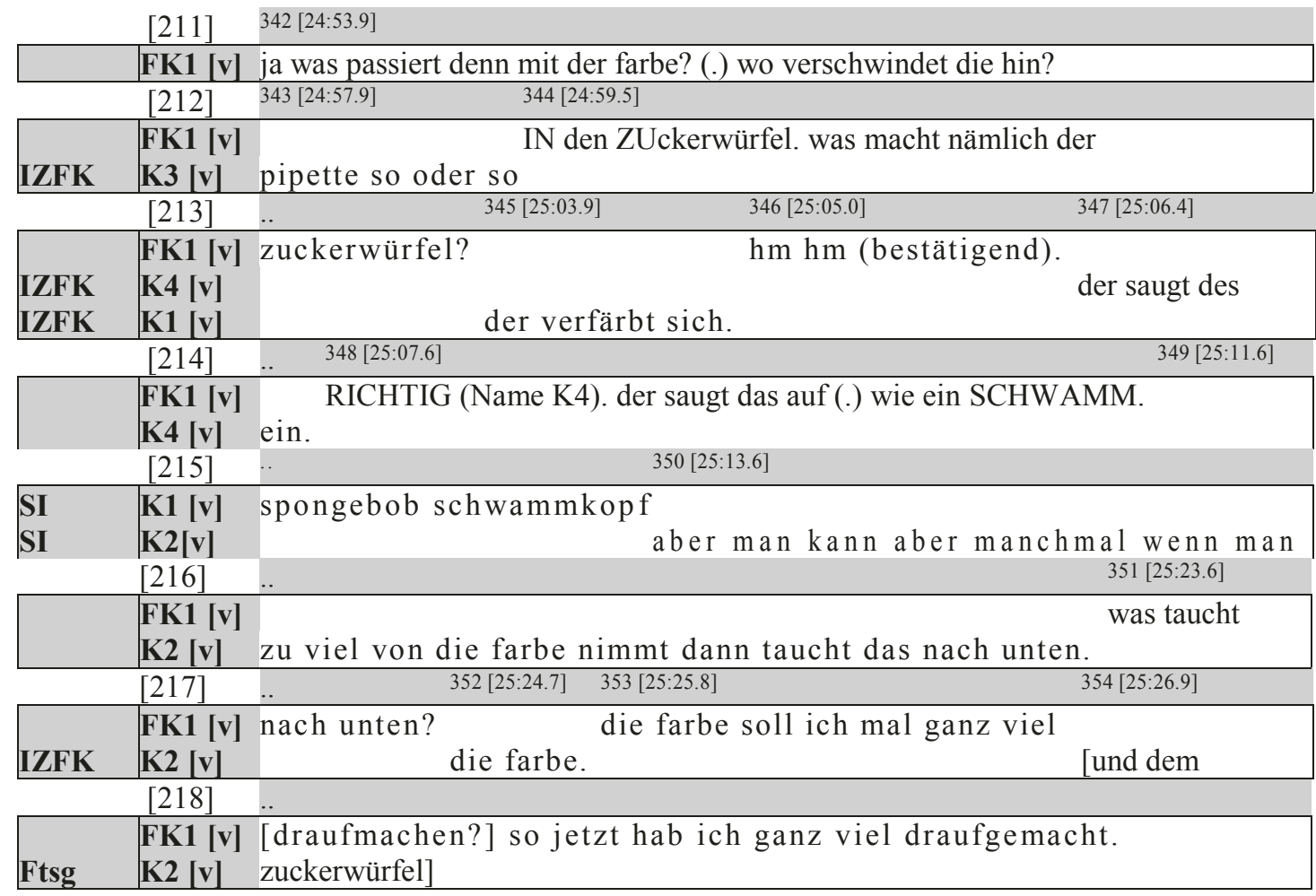

\section{Literatur}

Erdogan, E., Betz, T., Kämpfe, K., Kucharz, D., Mehlem, U. \& Rezagholinia, S. (o.J.). Dimensionen der Sprachförderung am Übergang Kindergarten-Grundschule. . In S. Gentrup, S. Henschel, K. Schotte, L. Beck, \& P. Stanat, (Hrsg.), Sprach- und Schriftsprachförderung gestalten: Evaluation von Qualität und Wirksamkeit umgesetzter Konzepte. Stuttgart: Kohlhammer.

F4transkript Version 5.40.0. (2015). Benutzerhandbuch f4transkript. Verfügbar unter: https://www.hwglu.de/fileadmin/user_upload/service/it-service-center/anleitungen/f4_manual_de.pdf [02.09.2019)

Hausendorf, H. \& Quasthoff, U. (2005). Sprachentwicklung und Interaktion. Eine linguistische Studie zum Erwerb von Diskursfähigkeiten. Radolfzell: Verlag für Gesprächsforschung.

Heller, V. (2012). Kommunikative Erfahrungen von Kindern in Familie und Unterricht: Passungen und Divergenzen. Tübingen: Stauffenburg.

Kucharz, D., Mehlem, U., Rezagholinia, S. \& Erdogan, E. (2018). Sprachförderung und sprachliche Entwicklung ein- und mehrsprachiger Kinder im letzten Kindergartenjahr. Empirische Pädagogik, 32(2), 177-197.

Kuckartz, U. (2010). Einführung in die computergestützte Analyse qualitativer Daten (3. Auflage). Wiesbaden: VS Verlag für Sozialwissenschaften.

MAXQDA (2018). MAXQDA 2018 Manual. Verfügbar unter: https://www.maxqda.de/download/Online-

Manual-Complete-Deutsch.pdf [02.09.2019]

Morek, M. (2012). Kinder erklären. Interaktionen in Familie und Unterricht im Vergleich. Tübingen: Stauffenburg.

Schegloff, E. (1996). Turn Organization: One Intersection of Grammar and Interaction, in Ochs, E., Schegloff,

E. A. \& Thompson, S. (Hrsg.). Interaction and Grammar (52-133). Cambridge: Cambridge University Press. 
Schmidt, T. (2016). EXMARaLDA Partitur-Editor Manual Version 1.6. Verfügbar unter: https://www.exmaralda.org/pdf/Partitur-Editor_Manual.pdf [02.09.2019]

Selting, M. (1995). Prosodie im Gespräch. Aspekte einer interaktionalen Phonologie der Konversation.

Tübingen: Niemeyer.

Selting, M., Auer, P., Barden, B., Bergmann, J., Couper-Kuhlen, E., Günthner, S., Meier, Ch., Quasthoff, U., Schlobinski, P. \& Uhmann, S. (1998). Gesprächsanalytisches Transkriptionssystem (GAT). Linguistische Berichte, 173, 91-122. 